\title{
Long-term study of water maser emission associated with young stellar objects
}

\section{The database}

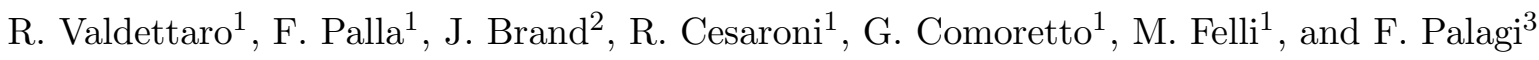 \\ 1 Osservatorio Astrofisico di Arcetri, Largo E. Fermi 5, 50125 Firenze, Italy \\ 2 Istituto di Radioastronomia CNR, Via Gobetti 101, 40129 Bologna, Italy \\ 3 C.A.I.S.M.I.-CNR, Largo E. Fermi 5, 50125 Firenze, Italy
}

Received 28 May 2001 / Accepted 15 November 2001

\begin{abstract}
We present the results of more than 10 years of monitoring of the water vapor maser emission in 14 star forming regions obtained with the Medicina 32-m radiotelescope. The sample of objects covers a large range of luminosities of the associated FIR sources. In order to present in a compact and representative way the large amount of data available, we give for each source: a time-velocity-flux density plot, the time dependent integrated flux, the lower and upper envelopes of the maser emission, the frequency of the maser occurrence as a function of velocity, and the mean velocity averaged over the observing period. A brief morphological description of the environment of the maser source is also given. The present data form the basis for a discussion of the main properties of the water vapor maser emission to be presented in a forthcoming paper.
\end{abstract}

Key words. masers - radio lines: interstellar - ISM: molecules

\section{Introduction}

Variability on short (days) and long (years) time scales is a well known property of $\mathrm{H}_{2} \mathrm{O}$ maser emission, associated either with Young Stellar Objects (YSOs) or with late-type stars. For example, from the comparison of the homogeneous set of observations of the Arcetri Atlas with literature data (Cesaroni et al. 1988), Comoretto et al. (1990) have shown that almost all $\mathrm{H}_{2} \mathrm{O}$ maser sources exhibit variations of the peak flux density as large as two orders of magnitude. Changes in the velocity of the peak emission has also been reported.

Emission variability in YSOs has been studied through single dish observations, paying attention mainly to the correlation (or lack thereof) between velocity components (S255, Cesaroni 1990), or to the change of the flux density, width and radial velocity of single components (Lekht 1995; Lekht et al. 1995; Márquez et al. 1998). These studies have supported the notion that $\mathrm{H}_{2} \mathrm{O}$ masers arise from instabilities in the shocked gas located in the circumstellar environment of the exciting YSO, either in a jet or in a disk.

The variability issue has also been studied from a statistical point of view for selected samples of sources. It

Send offprint requests to: R. Valdettaro,

e-mail: rv@arcetri.astro.it has been shown that the properties of the $\mathrm{H}_{2} \mathrm{O}$ maser emission depend on the FIR luminosity of the associated IRAS source, that there is a minimum luminosity $\left(\sim 25 L_{\odot}\right)$ above which maser emission should be present at some epoch. For low-luminosity objects the activity occurs for about one third of the embedded phase, i.e. when they are surrounded by large quantities of circumstellar dust that completely absorbs visible light from the YSO. The results indicate that the lower the source luminosity, the higher is the observed degree of variability of the $\mathrm{H}_{2} \mathrm{O}$ maser emission (e.g., Persi et al. 1994; Claussen et al. 1996; Valdettaro et al. 2001b).

In this paper, we set a basis for a more systematic study of the variability using a sample of 14 YSOs, covering a wide range of luminosities. We will describe some new procedures which are particularly useful for a morphological classification of the variability. We also give a full description of the observational results for each source. In a forthcoming paper, we shall present the analysis of the observed properties of $\mathrm{H}_{2} \mathrm{O}$ emission and variability of the present sample.

\section{The test sample}

To study the dependence of the variability of $\mathrm{H}_{2} \mathrm{O}$ masers on the far-infrared (FIR) luminosity (which is assumed to 
remain constant) of the associated YSO, a large sample of sources selected from the Arcetri Atlas has been monitored regularly since the beginning of the operating period of the Arcetri digital autocorrelator at the Medicina radiotelescope (1987), at a rate of $4-5$ observations per year. The sample was chosen using these criteria: a) the maser sources must be of the SFR-type, i.e. associated with Star Forming Regions (see for a detailed description Palagi et al. 1993; Valdettaro et al. 2001a); b) the associated IRAS sources should cover a range as large as possible of FIR luminosities; c) the sources are suitably distributed in right ascension so that they can be easily observed in a 24 hours run with a 5 min integration time on-source.

These constraints have resulted in a set of 44 sources. Since the observing program produces a large amount of data and the analysis requires well-tested procedures, we have selected 14 sources which are likely to be representative of the whole sample. The remaining 30 sources will be analyzed in a forthcoming study. The main properties of the 14 sources are given in Table 1 which lists: source names, coordinates, velocity of the molecular cloud relative to the LSR $\left(V_{\mathrm{cl}}\right)$, distance $(d)$, and integrated FIR luminosity $\left(L_{\mathrm{FIR}}\right)$. This latter quantity was derived by adopting the distance from Col. 7, assuming an emissivity proportional to frequency, correcting the observed IRAS flux densities for derived colour temperatures between two adjacent bands, and extrapolating the fluxes to 6 and $400 \mu \mathrm{m}$ (Wouterloot et al. 1995).

The test sample covers a range of FIR luminosities from $20 L_{\odot}$ to $1.8 \times 10^{6} L_{\odot}$. These values bracket the entire luminosity interval of the $\mathrm{H}_{2} \mathrm{O}$ maser sources of the SFRtype (Palagi et al. 1993). The validity of the SFR classification is also confirmed by other indicators (association with dense molecular cores, HiI regions, molecular outflows) which will be briefly discussed for each source. Of the 14 selected sources, association with an IRAS source is found in 13 cases, the exception being NGC 7129/FIRS2. Their location in the FIR color-color diagram is shown in Fig. 1. The dashed lines define the region occupied by sources with colors characteristic of ultracompact HiI regions (Wood \& Churchwell 1989). We see that all the sources fall inside or very close to these borders.

Ideally, from the observed maser variability, one should be able to infer the variability properties of the exciting YSO. However, while for $\mathrm{H}_{2} \mathrm{O}$ masers observed in evolved stars the association is unambiguous (i.e. there is only one powering star), for SFR-type masers the situation can be much more complex. Many YSOs, and consequently different groups of $\mathrm{H}_{2} \mathrm{O}$ masers, may be present in a small volume that fills our single dish beam of 1!9 HPBW (e.g., Forster \& Caswell 1989).

We have been careful to select sources from the Arcetri Atlas for which no other "single dish" maser has been reported in a region of several arcminutes, to eliminate confusion from nearby, but unrelated maser sources. However, we could not avoid complex structure on a scale smaller than our $H P B W$ and in some SFR this has been reported

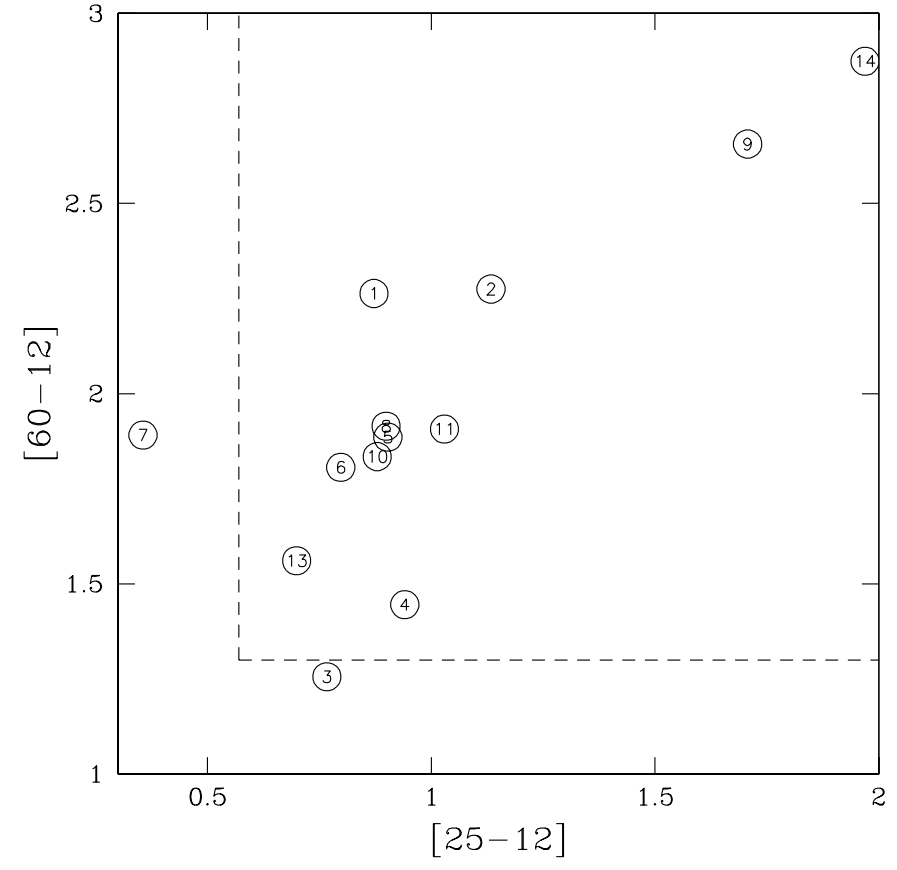

Fig. 1. Colour-colour diagram of the IRAS counterparts of the sources listed in Table 1. The inner box defines the boundaries of ultracompact Hil regions proposed by Wood \& Churchwell (1989).

during the course of our patrol. These cases will be pointed out in the description of the individual sources.

\section{Observations}

The present study is based on observations carried out with the Medicina $32-\mathrm{m}$ telescope ${ }^{1}(H P B W=1 ! 9)$ in the framework of a water maser monitoring program which is still going on. The time interval considered in this work goes from March 1987 to December 1999. During this period the whole system (antenna, receiver, autocorrelator) has been improved in various parts increasing the sensitivity and spectral resolution of the observations. A detailed description of the system and improvements can be found in Comoretto et al. (1990) and in Brand et al. (1994), respectively. In February 1997, the VLA-1 chip correlator was replaced by a new one based on the NFRA correlator chips and boards (Bos 1991). The new autocorrelator has 2048 delay channels and $160 \mathrm{MHz}$ maximum bandwidth in its standard configuration. In order to maintain compatibility with previous observations, we have continued to use only 1024 channels and a $10 \mathrm{MHz}$ bandwidth. Finally, owing to extensive maintenance works at the radiotelescope, there is a gap in the observations from April 1996 to February 1997.

The calibration process is of major concern for variability studies. Daily gain curves, which allow for

\footnotetext{
1 The Medicina VLBI radiotelescope is operated by the Radioastronomy Institute of the Italian National Research Council (CNR).
} 
Table 1. The $\mathrm{H}_{2} \mathrm{O}$ maser sample.

\begin{tabular}{|c|c|c|c|c|c|c|c|}
\hline$\#$ & $\begin{array}{l}\text { Source } \\
\text { Name }\end{array}$ & $\begin{array}{l}\text { Other name/ } \\
\text { Associated IRAS }\end{array}$ & $\begin{array}{c}\alpha(1950) \\
\text { h m s }\end{array}$ & $\begin{array}{c}\delta(1950) \\
\circ, \prime \prime\end{array}$ & $\begin{array}{c}V_{\mathrm{cl}}^{\mathbf{2}} \\
\left(\mathrm{km} \mathrm{s}^{-1}\right)\end{array}$ & $\begin{array}{l}d \\
(\mathrm{kpc})\end{array}$ & $\begin{array}{l}L_{\text {FIR }}^{\dagger} \\
\left(L_{\odot}\right)\end{array}$ \\
\hline 1 & Sh $2-184$ & $\begin{array}{l}\text { IRAS } 00494+5617 \\
\text { NGC } 281 \text { West }\end{array}$ & $00: 49: 28.6$ & $+56: 17: 33$ & $-30.8^{1}$ & $2.2^{6}$ & $7.9(3)$ \\
\hline 2 & L1455 IRS1 & IRAS $03245+3002$ & $03: 24: 34.0$ & $+30: 02: 36$ & $4.8^{2}$ & $0.35^{7}$ & $2.0(1)$ \\
\hline 3 & NGC 2071 & IRAS $05445+0020$ & $05: 44: 31.3$ & $+00: 20: 41$ & $9.5^{1}$ & $0.72^{8}$ & $1.4(3)$ \\
\hline 4 & Mon R2 IRS3 & $\begin{array}{l}\text { Mon R2 (2) } \\
\text { IRAS 06053-0622 }\end{array}$ & $06: 05: 21.7$ & $-06: 22: 35$ & $10.5^{1}$ & $0.8^{9}$ & $3.2(4)$ \\
\hline 5 & Sh 2-269 IRS2 & $\begin{array}{l}196.45-01.68 \\
\text { IRAS } 06117+1350\end{array}$ & $06: 11: 46.3$ & $+13: 50: 33$ & $18.2^{3}$ & $3.8^{6}$ & $6.0(4)$ \\
\hline 6 & W43 Main3 & $\begin{array}{l}32.82-0.06 \\
\text { IRAS } 18449-0158\end{array}$ & $18: 45: 11.0$ & $-01: 57: 57$ & $97.0^{1}$ & $7.3^{10}$ & $1.8(6)$ \\
\hline 7 & G32.74-0.08 & IRAS $18487-0015$ & $18: 48: 47.9$ & $-00: 15: 46$ & $38.2^{1}$ & $2.6^{10}$ & $5.3(3)$ \\
\hline 8 & G34.26+0.15 & $\begin{array}{l}\text { W } 44 \\
\text { IRAS } 18507+0110\end{array}$ & 18:50:46.3 & $+01: 11: 10$ & $57.8^{1}$ & $3.9^{10}$ & $7.5(5)$ \\
\hline 9 & G35.20-0.74 & IRAS $18556+0136$ & $18: 55: 40.7$ & $+01: 36: 30$ & $34.0^{1}$ & $1.8^{11}$ & $1.4(4)$ \\
\hline 10 & G59.78+0.06 & IRAS $19410+2336$ & $19: 41: 04.2$ & $+23: 36: 42$ & $22.3^{4}$ & $1.3^{11}$ & $5.3(3)$ \\
\hline 11 & Sh $2-128\left(\mathrm{H}_{2} \mathrm{O}\right)$ & $\begin{array}{l}\text { G97.53+3.19 } \\
\text { IRAS } 21306+5540\end{array}$ & $21: 30: 37.0$ & $+55: 40: 36$ & $-71.0^{4}$ & $6.5^{11}$ & $8.9(4)$ \\
\hline 12 & NGC 7129/FIRS2 & HH32-35 & $21: 41: 51.0$ & $+65: 49: 39$ & $-10.1^{1}$ & $1.0^{11}$ & $4.3(2)^{\ddagger}$ \\
\hline 13 & L1204-A & $\begin{array}{l}\text { Sh } 2-140 \text { IRS1 } \\
\text { IRAS } 22176+6303\end{array}$ & $22: 17: 41.2$ & $+63: 03: 43$ & $-7.1^{1}$ & $0.9^{6}$ & $2.6(4)$ \\
\hline 14 & L1204-G & IRAS $22198+6336$ & $22: 19: 49.8$ & $+63: 36: 27$ & $-10.8^{5}$ & $0.9^{6}$ & $5.8(2)$ \\
\hline
\end{tabular}

* Velocity of high-density gas $\left(\mathrm{NH}_{3}, \mathrm{CS}\right)$.

$\dagger$ Between brackets powers of 10 .

$\ddagger$ Luminosity from Eiroa et al. (1998).

Velocity references: 1. Anglada et al. (1996); 2. Juan et al. (1993); 3. Zinchenko et al. (1998); 4. Plume et al. (1992); 5. Tafalla et al. (1993).

Distance references: 6. (Spectro-)photometric distance from Brand \& Blitz (1993) compilation; 7. Herbig \& Jones (1983); 8. derived from Strom et al. (1974); 9. Racine (1968); 10. Near kinematic distance, using rotation curve of Brand \& Blitz (1993); 11. Kinematic distance, using observed velocity field of Brand \& Blitz (1993).

atmospheric attenuation and gravitational deformation of the primary mirror, are obtained through antenna temperature measurements at different elevations of the continuum source DR 21, whose flux density is assumed to be 18.8 Jy (Dent 1972). Antenna temperatures are derived from total power measurements in position switching mode. Integration time in each position was $10 \mathrm{~s}$ with $400 \mathrm{MHz}$ bandwidth. The zenith system temperature is about $120 \mathrm{~K}$ in clear weather conditions. For each observing day, all gain measurements as a function of elevation are fitted with a polynomial curve, which is then used to calculate the conversion factor from antenna temperature to flux density for the spectra observed on that day. The calibration error resulting from the dispersion of the single measurements around the fit is found to be $19 \%$. On a few dates, no gain curve was measured: in these cases we have applied the gain curve closest in time and we estimate a corresponding calibration uncertainty of $7 \%$. Therefore, we can estimate an average calibration error of $20 \%$.
To reduce the effects of atmospheric attenuation variations, observations were obtained in good weather conditions and, whenever possible, around source meridian transit time. In order to test the reliability of our procedure, we have compared our results with those available in the literature always finding a very good agreement. As an example, Fig. 2 displays the peak flux density versus time for the source L1455 IRS1. This source was observed independently by Claussen et al. (1996) in an interval of time partially overlapping our observations (Fig. 2, triangles). Apart from an episode of strong and rapid variability (at about 1800 days) not unusual for $\mathrm{H}_{2} \mathrm{O}$ masers, the two sets of measurements agree well, confirming the quality of our observations and calibration procedures.

\section{General properties of the maser emission}

The amount of information collected during the observations is very large and a concise way of data presentation is important for a visual appreciation of the properties of 


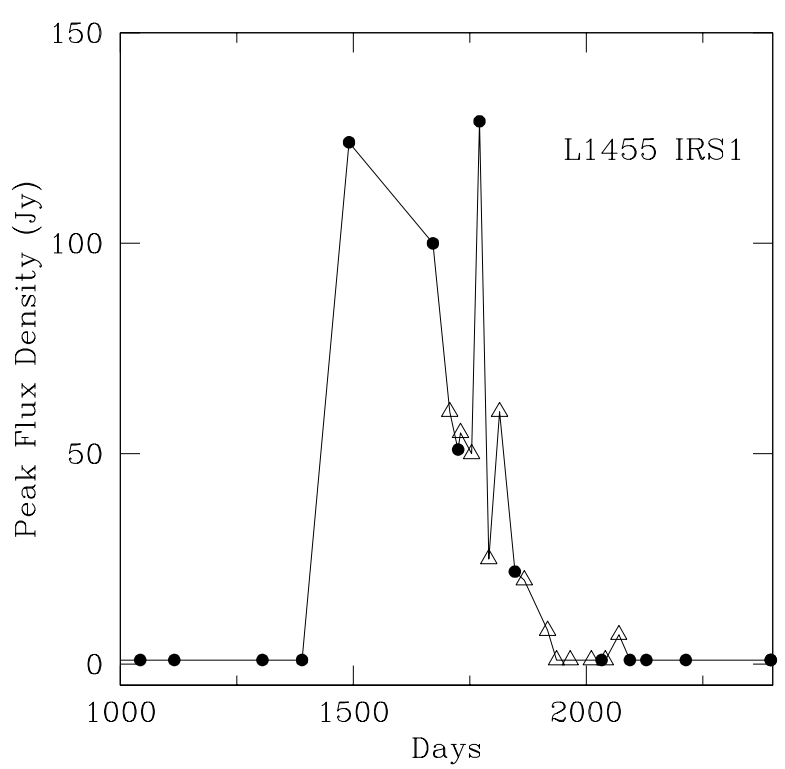

Fig. 2. Time variation of the peak flux of L1455 IRS1 in a restricted time interval. The horizontal axis gives the days elapsed since the epoch of the first observation (30/03/1987). Filled circles are our observations, while triangles display the monthly epoch observations of Claussen et al. (1996).

maser variability over a long time interval. In the literature, data are often presented as time sequence of spectra (Liljeström et al. 1989; Claussen et al. 1996; Lekht et al. 1999), or as plots of derived quantities, such as peak flux density, velocity of the emission, and linewidths as a function of time. However, neither of these methods seem to be fully adequate to highlight the evolutionary features of the observed velocity components in a synthetic form. A better visualization of the time variation of the maser emission can be obtained by showing the flux density versus velocity and time in the same diagram (e.g., Lekht et al. 1999).

To build these plots, observations separated by less than ten days have been averaged, so variations on time scales shorter than 10 days are filtered out. Since the observed data are not distributed evenly in time, some kind of interpolation between two adjacent observations must be used. As no assumption can be made on the evolution of the $\mathrm{H}_{2} \mathrm{O}$ maser components, we have chosen a linear interpolation. This procedure produces an artificial increase in the lifetime of features, particularly when long time intervals between two consecutive observations occur.

The display of the peak or integrated flux densities is often used to analyze the light curve of the maser emission. The peak flux density is useful only if one major component is present throughout the entire observing period. It has less physical meaning for complex spectra with multiple components. In this case, the use of the integrated flux densities is preferable.

Another interesting quantity that describes the degree of maser variability can be obtained by plotting the upper and lower envelopes of the spectra over the whole period of observation. The upper envelope describes the maximum flux density reached at each velocity, and gives an overall view of the entire velocity interval of the maser emission. Its integral and the derived luminosity, $L_{\mathrm{H}_{2} \mathrm{O}}^{*}$, represent the maximum emission that can be emitted by the source if all the velocity components were to emit at their maximum level and at the same time. On the other hand, the lower envelope identifies the components that are always active. When the lower envelope is zero over the whole velocity interval, it indicates that no velocity component is constantly present during the observing period, but it does not necessarily imply that the maser is quiescent at some epoch over the entire velocity range.

Finally, to determine the velocity components which are more frequently present in the spectra (independently of their intensity), we present the frequency of occurrence of maser emission above the $5 \sigma$ noise at a given velocity. To produce this plot we have binned the total velocity interval in a number of velocity channels (usually $\Delta V \simeq 0.3 \mathrm{~km} \mathrm{~s}^{-1}$ ) and set a counter for each channel that increases by one unit every time the flux density in the channel is greater than the $5 \sigma$ noise of the spectrum.

The velocities of the $\mathrm{H}_{2} \mathrm{O}$ masers are often compared with those of the molecular clouds in which they are embedded. The results indicate a good general agreement, but with a relatively large scatter of $\simeq 7-10 \mathrm{~km} \mathrm{~s}^{-1}$ (Wouterloot et al. 1995; Anglada et al. 1996). Probably, this large scatter comes from the fact that one compares an instantaneous maser spectrum with the velocity of the molecular gas and is forced to choose, for instance, the velocity of the maser component with the highest intensity. Given our larger time coverage of the variable maser emission, we have computed for each source two mean velocities: 1) the velocity obtained from the expression

$V_{\mathrm{up}}=\frac{\int V F_{\nu}^{\mathrm{up}} \mathrm{d} V}{\int F_{\nu}^{\mathrm{up}} \mathrm{d} V}$

where $F_{\nu}^{u p}$ is the flux of the upper envelope spectrum; 2) the velocity obtained from the expression

$V_{\mathrm{fr}}=\frac{\int V \mathcal{N} \mathrm{d} V}{\int \mathcal{N} \mathrm{d} V}$

where $\mathcal{N}$ is the frequency of occurrence, namely the number of times that emission is detected at each velocity in the spectrum. These two velocities are useful to separate red- and blue-shifted velocity features in a consistent way. In addition, we have computed the dispersions associated with these velocities, called respectively $\Delta V_{\mathrm{up}}$ and $\Delta V_{\mathrm{fr}}$. The derived quantities $V_{\mathrm{fr}}, \Delta V_{\mathrm{fr}}, V_{\mathrm{up}}$, and $\Delta V_{\mathrm{up}}$, are given for each source in Table 2, along with the integrated flux of the upper envelope and the corresponding maximum maser luminosity $L_{\mathrm{H}_{2} \mathrm{O}}^{*}$.

In studies of maser variability, it is a common procedure to separate steady, long-lasting features from outbursting ones which show at time $t_{\text {initial }}$ a rapid increase of the flux above $F_{\text {steady }}$, up to a maximum $F_{\text {peak }}$ followed by a rapid return to $F_{\text {steady }}$ (at time $t_{\text {final }}$ ). Quantities such as $\Delta F \equiv\left(F_{\text {peak }}-F_{\text {steady }}\right)$ and $\Delta t \equiv\left(t_{\text {final }}-t_{\text {initial }}\right)$ are 
Table 2. Main parameters of the $\mathrm{H}_{2} \mathrm{O}$ maser emission.

\begin{tabular}{lrrrrrc}
\hline \hline$\#$ & $V_{\text {fr }}$ & $\Delta V_{\text {fr }}$ & $V_{\text {up }}$ & $\Delta V_{\text {up }}$ & Int. Fl. & $L_{\mathrm{H}_{2} \mathrm{O}}^{*}$ \\
& $(1)$ & $(2)$ & $(3)$ & $(4)$ & $(5)$ & $(6)$ \\
\hline 1 & -31.4 & 12.2 & -32.2 & 12.1 & 1770 & $2.0(-4)$ \\
2 & 4.3 & 4.1 & 4.1 & 4.1 & 340 & $9.6(-7)$ \\
3 & 10.3 & 15.7 & 12.1 & 10.4 & 26070 & $3.1(-4)$ \\
4 & 10.6 & 11.3 & 10.5 & 9.3 & 1600 & $2.4(-5)$ \\
5 & 17.8 & 7.2 & 17.5 & 7.9 & 800 & $2.7(-4)$ \\
6 & 101.8 & 31.4 & 101.2 & 28.5 & 6340 & $7.8(-3)$ \\
7 & 33.7 & 5.7 & 34.1 & 7.1 & 110 & $1.7(-5)$ \\
8 & 51.6 & 26.2 & 54.2 & 25.8 & 8260 & $2.9(-3)$ \\
9 & 33.8 & 9.1 & 34.2 & 8.0 & 1010 & $7.6(-5)$ \\
10 & 24.3 & 12.9 & 26.0 & 10.4 & 1600 & $6.2(-5)$ \\
11 & -74.1 & 11.9 & -72.7 & 13.3 & 3980 & $3.9(-3)$ \\
12 & -5.2 & 14.2 & -4.6 & 12.6 & 2950 & $6.8(-5)$ \\
13 & -6.3 & 22.0 & -8.5 & 20.0 & 1340 & $2.5(-5)$ \\
14 & -17.1 & 14.8 & -18.1 & 14.3 & 1210 & $2.3(-5)$ \\
\hline
\end{tabular}

Columns 1 to 4 : $\mathrm{km} \mathrm{s}^{-1}$; Col. 5: Jy km s${ }^{-1}$; Col. $6: L_{\odot}$; the number in parenthesis is the exponent of 10 .

used to define the burst features (e.g., Liljeström \& Gwinn 2000). Some indications of the values of $\Delta F$ and $\Delta t$ will be given in the presentation of the sources, but the general discussion of their properties is deferred to the paper with the analysis.

Of course, the details contained in each spectrum are by far greater than the information that can be tabulated or plotted in our figures. For those interested, the calibrated and reduced spectra can be obtained upon request from palagi@arcetri.astro.it.

\section{Presentation of the individual sources}

\subsection{Sh $2-184$}

\subsubsection{The maser environment}

Sh 2-184 (also known as NGC 281) is a bright diffuse HII region associated with a molecular cloud in the outer Galaxy. The molecular cloud is associated with IRAS 00494+5617, an $\mathrm{NH}_{3}$ core, an $\mathrm{H}_{2} \mathrm{O}$ maser and $1.3 \mathrm{~mm}$ continuum dust peak.

High resolution maps in $\mathrm{C}^{18} \mathrm{O}(1 \rightarrow 0), \mathrm{C}^{18} \mathrm{O}(2 \rightarrow 1)$, $\mathrm{C}^{34} \mathrm{~S}(3 \rightarrow 2)$ (Megeath \& Wilson 1997) show that Sh 2-184 is structured into three clumps, two in the northern part, named NE and NW, and the third (S) in the southern part. The morphological relationship between the diffuse $\mathrm{H} \alpha$-emitting gas of Sh $2-184$, the radio continuum emission of the densest part of the HII region and the molecular clouds is best explained by Fig. 5 of Megeath \& Wilson (1997). The diffuse radio HII region is located between the HII region and the molecular cloud and probably represents the effect of the ionizing radiation of the OB star in the Sh 2-184 nebula on the outer part of the molecular cloud. The $\mathrm{H}_{2} \mathrm{O}$ maser is located in the NW molecular cloud and coincides with the $1.3 \mathrm{~mm}$ dust peak.

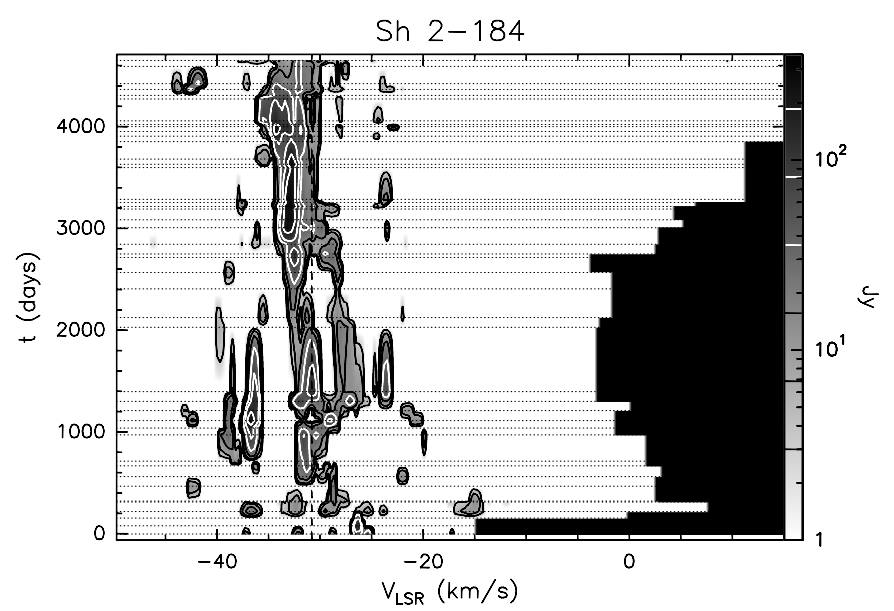

Fig. 3. Grey scale plot and contour map of the line flux density versus velocity as a function of time for Sh 2-184. The values of the contour levels are indicated by the horizontal lines in the grey-scale wedge to the right of the map. The dotted horizontal lines correspond to the dates of the observed spectra. The vertical dashed line indicates the systemic velocity of the associated molecular cloud $V_{\mathrm{cl}}$, as given in Table 1 . The black portion of the plot is the unobserved part of the spectrum.

The molecular line emission intensity peaks at $V_{\mathrm{LSR}}=$ $-32 \mathrm{~km} \mathrm{~s}^{-1}$ for the NE and NW clumps, while in the S clump the peak occurs at $V_{\mathrm{LSR}}=-29 \mathrm{~km} \mathrm{~s}^{-1}$, indicating that it is spatially and kinematically distinct from the other clouds (Megeath \& Wilson 1997).

A VLA map at $22 \mathrm{GHz}$ (Tofani et al. 1995) separates the $\mathrm{H}_{2} \mathrm{O}$ maser emission into three components, all of them in the NW clump and with maximum separation of $\sim 8^{\prime \prime}$ (i.e. well within the Medicina beam). No radio continuum ultracompact (UC) HII region was found near the position of the $\mathrm{H}_{2} \mathrm{O}$ components. Two of the $\mathrm{H}_{2} \mathrm{O}$ maser components show emission only at $V_{\mathrm{LSR}}=-28 \mathrm{~km} \mathrm{~s}^{-1}$ while the third component has emission ranging from $V_{\mathrm{LSR}}=-40 \mathrm{~km} \mathrm{~s}^{-1}$ to $V_{\mathrm{LSR}}=-22 \mathrm{~km} \mathrm{~s}^{-1}$.

\subsubsection{Water maser properties}

The database consists of 47 spectra extending over a period of 4654 days. Figure 3 shows a weak anticorrelation between the maximum intensity and the emission range, i.e. when the peak emission tends to increase (even if in a discontinuous way), the emission range tends to contract from $V_{\mathrm{LSR}}=(-43 \div-15) \mathrm{km} \mathrm{s}^{-1}$ at the beginning of the observing period, to $V_{\mathrm{LSR}}=(-36 \div-24) \mathrm{km} \mathrm{s}^{-1}$ at its end.

There is one component showing a velocity drift: $V_{1} \sim$ $-32 \mathrm{~km} \mathrm{~s}^{-1}$ with $t_{\text {initial }}=600$ days, and $t_{\text {final }}=1400$ days. A global velocity drift of the emission toward the blue is also evident in Fig. 3. Bursts of weak intensity $(\Delta F \simeq$ $10 \mathrm{Jy})$ and short duration ( $\Delta t \simeq 150$ days) are more frequent at large velocity offsets from the cloud velocity while the emission close to the cloud velocity is more steady with bursts of longer duration ( $\Delta t \simeq 1000$ days) and greater 

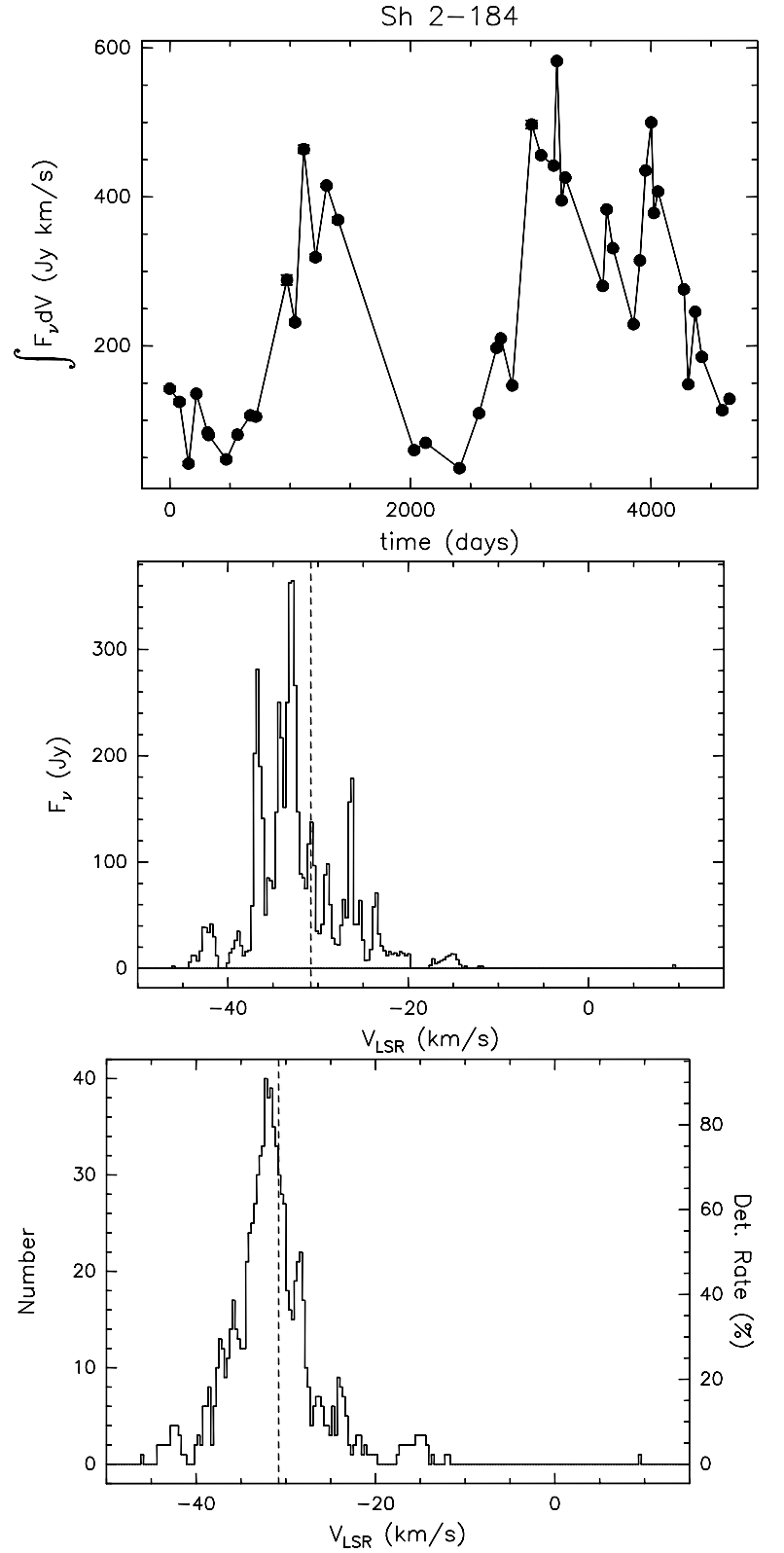

Fig. 4. Source Sh 2-184. Top panel: integral of the flux density over the observed velocity range as a function of time; empty circles (if any) indicate upper limits. Middle panel: upper and lower envelopes of the flux density measured during our monitoring (see text for details). Bottom panel: frequency of occurrence of the maser emission in the observed velocity range: the left axis gives the number of times the maser emission was detected above $5 \sigma$ at each velocity; the right axis reports the corresponding detection rate. All histograms are binned with $0.3 \mathrm{~km} \mathrm{~s}^{-1}$ resolution; the vertical dashed lines corresponds to the systemic velocity of the associated molecular cloud, $V_{\mathrm{cl}}$, as given in Table 1 .

intensity $(\Delta F \simeq 300 \mathrm{Jy})$. As displayed in Fig. 4 , the lower envelope of the emission is zero over the entire velocity range, i.e. there is no velocity component which is always present. The plot of the integrated emission (see Fig. 4) may suggest a periodicity of the order of 2000 days, but the data base is just too short to justify a more definite statement.

\subsection{L1455 IRS1}

\subsubsection{The maser environment}

The $\mathrm{H}_{2} \mathrm{O}$ maser is located in the dark cloud L1455 at the western edge of the Perseus cloud complex. There are three IRAS sources in the cloud. L1455 IRS1, also known as IRAS $03245+3002$, is the most luminous one $\left(L_{\mathrm{bol}}=20 L_{\odot}\right)$, undetected both in the optical and near-infrared wavelengths. It is located $\sim 2^{\prime}$ northwest of L1445 IRS2 (IRAS 03247+3001), an H $\alpha$ emission line star which appears as a faint, red nebulous object (Cohen 1980). Finally, the third embedded source is L1445 IRS3 (IRAS $03249+2957$ ), located $\sim 5^{\prime}$ farther to the southeast of IRS1, with a luminosity $L_{\text {bol }}<0.5 L_{\odot}$. $\mathrm{H}_{2} \mathrm{O}$ maser emission was found only in association with L1455 IRS1.

Moderately high angular resolution molecular emission maps (Bachiller \& Cernicharo 1986; Juan et al. 1993) have shown that the L1455 core consists of two main components at $V_{\mathrm{LSR}}=+4.8$ and $5.5 \mathrm{~km} \mathrm{~s}^{-1}$, respectively, plus several other condensations with individual masses ranging from 1 to $10 M_{\odot}$. From the enhancements of the widths of CS and $\mathrm{NH}_{3}$ lines, Juan et al. (1993) argue that the cloud at $V_{\mathrm{LSR}}=+4.8 \mathrm{~km} \mathrm{~s}^{-1}$, associated with L1455 IRS1, is connected with the CO outflows detected in the region (Frerking \& Langer 1982; Goldsmith et al. 1984; Levreault 1988).

Recent optical, near-infrared and submm high resolution observations of L1455 IRS1 have revealed the actual geometry of the region (Davis et al. 1997a,b; Bally et al. 1997), confirming that the bipolar outflow is driven by this source. The peaks in the $\mathrm{CO}$ outflow lobes coincide spatially with compact $\mathrm{H}_{2}$ line emission knots. Also, [SII] $\lambda \lambda 6716,6713 \AA$ images reveal a close association between the collimated $\mathrm{CO}$ outflow and the underlying stellar jet. Davis et al. (1997a,b) present evidence for a jet-driven outflow, dominated by prompt entrainment of molecular material.

Bally et al. (1997) have detected five Herbig-Haro objects in the vicinity of L1455, three of them likely powered by L1455 IRS1. No continuum centimeter radio emission greater than $0.1 \mathrm{mJy}$ was found with the VLA at the position of the source (Meehan et al. 1998). The $2 \mu \mathrm{m}$ source which corresponds to L1455 IRS1 (Ladd et al. 1993; Hodapp 1994; Persi et al. 1994; Tapia et al. 1997) has a spectral energy distribution typical of an extreme Class I object.

\subsubsection{Water maser properties}

Figure 5 shows the velocity-time contour plot of the Medicina observations. The database consists of 48 spectra, extending over a period of 4644 days. The source has not been observed at all in 1988. Starting in 1990, we have taken between 3 and 6 spectra per year, with an average 


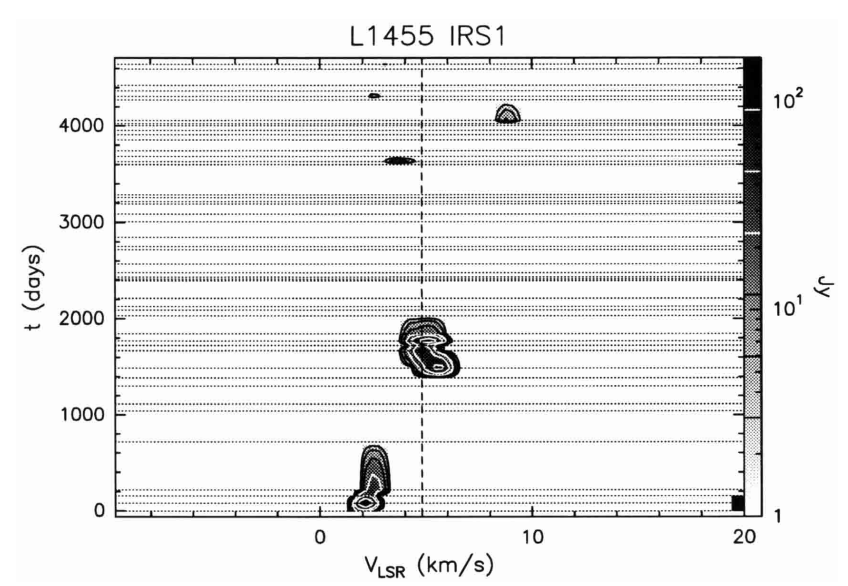

Fig. 5. Same as Fig. 3 for L1455 IRS1.
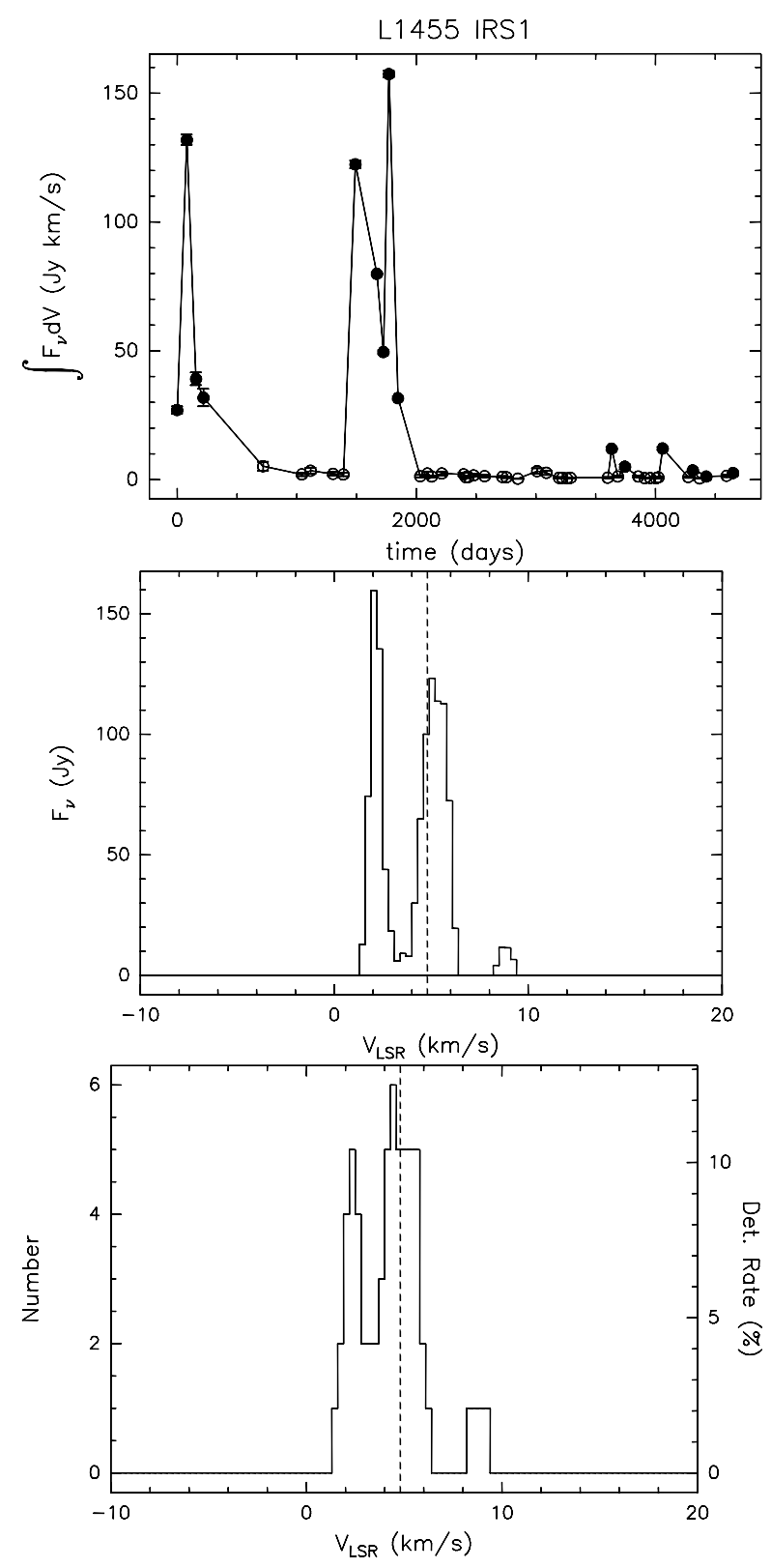

Fig. 6. Same as Fig. 4 for L1455 IRS1.

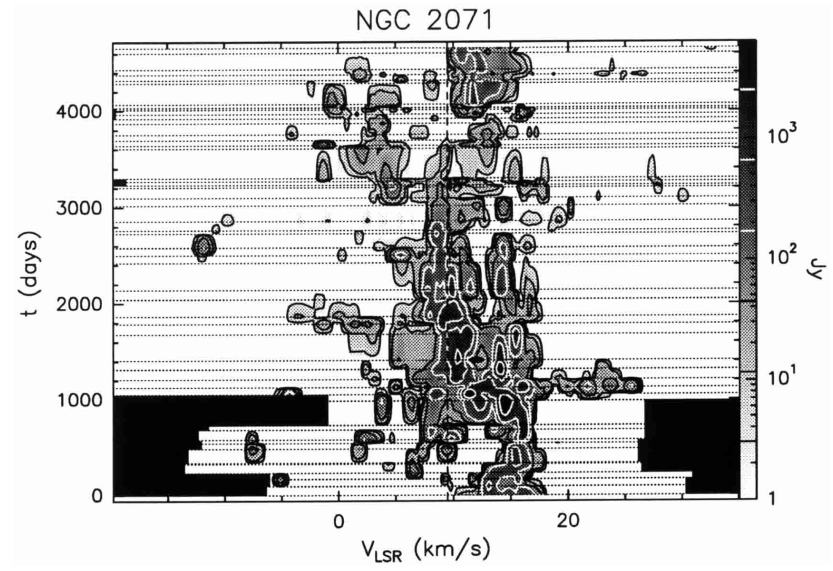

Fig. 7. Same as Fig. 3 for NGC 2071.

time interval of 2.7 months. As pointed out in Sect. 3, the peak flux density agrees very well with the observations of Claussen et al. (1996) for the one year period of overlap (1992); the same is true for the velocity of the maser.

Maser emission is highly episodic: there are only six events separated by extended periods of quiescence. Owing to the absence of observations in 1988, we do not know exactly when the maser first became quiescent during our monitoring campaign.

For L1455 IRS1, since the emission emerges from the noise, $\Delta F \geq\left(F_{\text {peak }}-F_{\text {noise }}\right)$ can be as high as $170 \mathrm{Jy}$, while $\Delta t$ varies from $\sim 50$ days to up to $\sim 300$ days.

The peak of the emission occurs each time at a different velocity, varying from $2 \mathrm{~km} \mathrm{~s}^{-1}$ to $5.5 \mathrm{~km} \mathrm{~s}^{-1}$ to $9 \mathrm{~km} \mathrm{~s}^{-1}$. Is this the evidence of a continuous velocity drift of the same physical structure or episodic masing of independent structures? In the former case, the velocity drift is $\simeq 0.6 \mathrm{~km} \mathrm{~s}^{-1} \mathrm{yr}^{-1}$, similar to that found in W75N (Hunter et al. 1994). The second possibility is supported by the wiggling of the jet that powers the outflow (Davis 1997b).

From their sample of masers associated with low-mass stars, Claussen et al. (1996) infer that in this range of luminosities the maser is active (i.e., above the noise level) for about $1 / 3$ of the time. With the inclusion of our observations, the fraction of maser activity in this source may be even smaller.

Figure 6 displays the upper and lower envelopes of maser emission as a function of velocity. Given the short periods of activity and the change in velocity, this is simply the sum of the four independent features. The lower envelope is equal to zero over the entire velocity range.

Figure 6 presents the histogram of the frequency of occurrence of the maser features as a function of velocity. The two features close to $V_{\mathrm{cl}}$ are the most frequent. 


\subsection{NGC 2071}

\subsubsection{The maser environment}

The $\mathrm{H}_{2} \mathrm{O}$ maser emission comes from an intermediate luminosity SFR located in a large molecular cloud (Calamai et al. 1982; Snell et al. 1984; Torrelles et al. 1998) about $4^{\prime}$ north of the reflection nebula NGC 2071.

A cluster of near-IR sources has been detected in a region of size $\sim 20^{\prime \prime}$ (Persson et al. 1981; Walther et al. 1991), some of which were also detected in the radio continuum as compact Hiı regions (Calamai et al. 1982; Snell \& Bally 1986; Tofani et al. 1995; Torrelles et al. 1998). A bipolar molecular outflow in this area has been seen in $\mathrm{CO}, \mathrm{SO}$, $\mathrm{SiO}$ and $\mathrm{HCO}^{+}$(Snell et al. 1984; Moriarty-Schieven et al. 1989; Chernin \& Masson 1992, 1993; Girart et al. 1999). It is oriented in the NE-SW direction with a maximum extension of $3^{\prime}$ in each direction.

High-resolution VLA radio observations in 1983 have detected water maser emission from two of the three nearIR and radio components (NGC 2071/IRS1/IRS3, Sandell et al. 1985). In Tofani et al. (1995) only IRS1 has associated maser emission, while Torrelles et al. (1998) find emission from IRS1 and IRS3. The distribution of the $\mathrm{H}_{2} \mathrm{O}$ spatial components in IRS1 (6 spots in Tofani et al. 1995, 22 spots in Torrelles et al. 1998), which is the brightest $10 \mu \mathrm{m}$ source and the suggested source powering the bipolar molecular outflow, traces at scales of $300 \mathrm{AU}$ the large-scale $\mathrm{H}_{2}$ outflow observed at a few thousands $\mathrm{AU}$ from the exciting source (Lane \& Bally 1986; Burton et al. 1989; Garden et al. 1990; Aspin et al. 1992; Torrelles et al. 1998). The velocities of the 22 spots are between 0.1 and $23.2 \mathrm{~km} \mathrm{~s}^{-1}$. The $\mathrm{H}_{2} \mathrm{O}$ masers in IRS3 (13 spots in Torreles et al. 1998) are tightly packed in a disk $40 \mathrm{AU}$ in size, almost perpendicular to the major axis of a radio continuum jet, possibly located in a rotating protoplanetary disk of $20 \mathrm{AU}$ radius. The velocities of the 13 spots are between -1.9 and $17.2 \mathrm{~km} \mathrm{~s}^{-1}$.

Strong variability of the $\mathrm{H}_{2} \mathrm{O}$ maser emission from this SFR is documented since 1977 (Pankonin et al. 1977). The emission has already been studied extensively with single dish observations during 1979-1981 by Lekht et al. (1982) and during $1982-1993$ by Berulis et al. (1996). The ample overlap (from 1987 to 1993) with our patrol allows a detailed comparison between the different sets of observations: the evolution of individual velocity components shows a very good agreement.

\subsubsection{Water maser properties}

The database consists of 50 spectra, extending over a period of 4658 days. Starting in 1987, we have acquired between 2 and 6 spectra per year, with an average time interval of 3 months. Figure 7 shows the velocity-time contour plot of the Medicina observations. The situation is indeed very complex, with spectra showing more than 10 distinct velocity components. Bursts of emission are visible all over the observed velocity range $\left(-20\right.$ to $\left.30 \mathrm{~km} \mathrm{~s}^{-1}\right)$ and at all times, probably the result of the three different centres of activity within the SFR. This fact is confirmed by the time plot of the integrated flux density (see Fig. 8) whose value is always greater than zero.

All the individual emission bursts seem to be of relatively short duration, and the observed pattern could be described as the superposition of many individual small duration bursts at random velocities rather than as the weakly varying emission of the same velocity component. In fact, no velocity feature is constantly present during our patrol, as shown in Fig. 8 where the upper and lower envelopes of the maser emission are plotted: the lower envelope is equal to zero over the whole observed velocity range. As already mentioned, this does not mean that there is one epoch in which the entire maser source is quiescent, but that during the 12 years of our patrol there are always velocity intervals in which there is no emission, and that on a long time scale the non-emission velocity intervals cover the entire velocity range.

We note that $\Delta F$ can be as high as 6000 Jy (see also Berulis et al. 1996) and is usually much stronger near $V_{\mathrm{cl}}$ than on the red and blue extremes, where values of the order of few Jy are more common. This effect is also indicated by the upper envelope of Fig. 8 where the highest peaks are at $\sim 11$ and $14 \mathrm{~km} \mathrm{~s}^{-1}$.

The time extent of the bursts can be up to 1000 days, even though $\Delta t$ of the order of the sampling interval ( $\sim 100$ days) are more typical. The four bursts described by Berulis et al. (1996) with more frequent observations than ours have duration between 90 and 150 days. The duration appears to be longer for velocities closer to $V_{\mathrm{cl}}$ than towards the red and blue extremes. Both of these results are in good agreement with those found in the more luminous source $\mathrm{W} 49 \mathrm{~N}$ on a much larger velocity range (Liljeström \& Gwinn 2000) which indicate that $\Delta t(V)$ and $\Delta F(V)$ are maximum close to the cloud velocity.

Figure 8 presents the histogram of the frequency of occurrence of the maser features as a function of velocity. The bulk of the activity is between 0 and $20 \mathrm{kms}^{-1}$, and the distribution is practically flat between 7 and $17 \mathrm{kms}^{-1}$.

A couple of velocity drifts are evident in Fig. 7 (for example, for $600<t<1200$ and $12<V_{\mathrm{LSR}}<17 \mathrm{~km} \mathrm{~s}^{-1}$, and for $1200<t<2800$ and $8<V_{\mathrm{LSR}}<12 \mathrm{~km} \mathrm{~s}^{-1}$ ), but others can be found from a more accurate analysis of the spectra. Velocity drifts of $1-2 \mathrm{~km} \mathrm{~s}^{-1} \mathrm{yr}^{-1}$ were also reported by Berulis et al. (1996) for the four burst examined. However, given the peculiar bursting nature of the emission in this source and the large number of spatial components, some confusion could arise by the superposition of physically unrelated bursts. In summary, velocity drifts of single physical components seem to be quite common, but the detailed study of this particular effect is rather difficult owing to the large number of components and to our coarse time sampling compared with the typical duration of a single burst. 

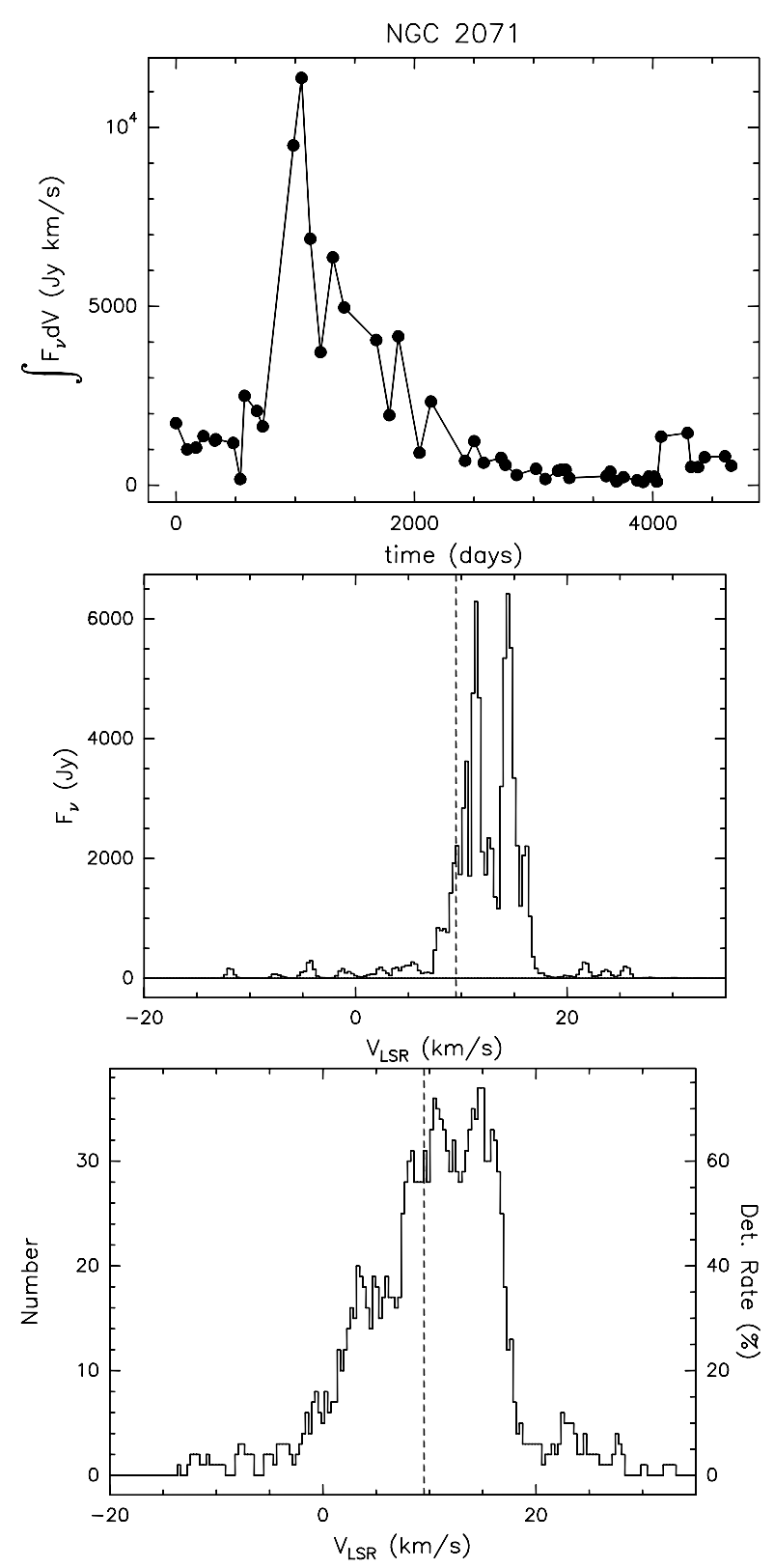

Fig. 8. Same as Fig. 4 for NGC 2071.

\subsection{Mon R2 IRS3}

\subsubsection{The maser environment}

The maser is within the Mon R2 star-forming region. The brightest IR sources sources are IRS1, IRS2 and IRS3 (Beckwith et al. 1976; Howard et al. 1994) with a total luminosity of about $5 \times 10^{4} L_{\odot}$ (Thronson et al. 1980). IRS1 $\left(\alpha_{1950}=06^{\mathrm{h}} 05^{\mathrm{m}} 20^{\mathrm{s}} ; \delta_{1950}=-06^{\circ} 22^{\prime} 35^{\prime \prime}\right)$ is a B0 star, exciting a blister type HiI region (Gilmore 1980; Massi et al. 1985). IRS2 $\left(\alpha_{1950}=06^{\mathrm{h}} 05^{\mathrm{m}} 19.5^{\mathrm{s}} ; \delta_{1950}=\right.$ $-06^{\circ} 22^{\prime} 25^{\prime \prime}$ ) illuminates a reflection nebula (Hodapp 1987; Aspin \& Walther 1990), while IRS3 $\left(\alpha_{1950}=06^{\mathrm{h}} 25^{\mathrm{m}} 21.8^{\mathrm{s}}\right.$; $\left.\delta_{1950}=-06^{\circ} 22^{\prime} 25^{\prime \prime}\right)$ is the brightest FIR source, offset by $\left(22^{\prime \prime} 4,133^{\prime \prime} 4\right)$ with respect to the position of the VLA $6 \mathrm{~cm}$ HiI region and outside its sharp boundary

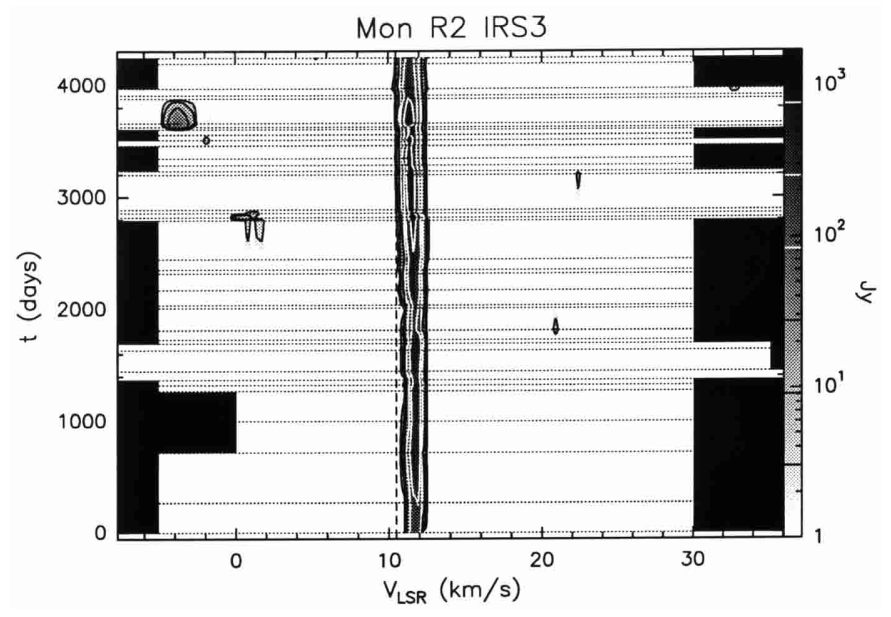

Fig. 9. Same as Fig. 3 for Mon R2 IRS3.

(Massi et al. 1985). On morphological grounds, IRS3 should be in an evolutionary stage earlier than IRS1.

The three infrared sources are but the brightest objects of a large population of embedded sources detected in a large-scale $K$-band image (Aspin \& Walther 1990). Recently, Carpenter et al. (1997) have found that the infrared cluster extends over a $\sim 1.1 \times 2.1 \mathrm{pc}$ area with a FHWM size of $\sim 0.38 \mathrm{pc}$ and that it contains at least 300 stars (stellar volume density $\sim 9000 \mathrm{stars}^{-3} \mathrm{c}^{-3}$ ). In many respects, the cluster is similar to other young clusters, such as NGC 2024 and the Orion cluster.

VLA observations in the $\mathrm{H}_{2} \mathrm{O}$ line and $8.4 \mathrm{GHz}$ continuum (Tofani et al. 1995) show that the maser emission comes from a single spot close to IRS3. No radio continuum emission above $0.3 \mathrm{mJy}$ from an unresolved component is associated with either the maser or IRS3.

The properties of the molecular gas associated with Mon R2 have been studied by many authors after the initial detection by Kutner \& Tucker (1975) and Loren et al. (1977). The most conspicuous feature is the presence of a large outflow (extending over $25^{\prime}, \sim 7 \mathrm{pc}$ ), containing $\sim 200 M_{\odot}$ of accelerated gas (Loren 1981; Wolf et al. 1990). Recent high-resolution studies of the CS and ${ }^{13} \mathrm{CO}$ emission of the central part of the outflow have revealed the existence of several clumps moving with the flow and containing several solar masses of gas each. The emission of the dense CS gas presents shell-like features with the IR cluster at their apex, resembling those found in the L1551 and NGC 2071 outflows (Tafalla et al. 1994, 1997). The source exciting this extreme outflow is still not identified. Giannakopoulou et al. (1997), in an attempt to locate the source driving the outflow, have detected high-velocity $\mathrm{CO}$ gas $\left(\Delta V_{\mathrm{CO}} \simeq 75 \mathrm{~km} \mathrm{~s}^{-1}\right)$ toward IRS3, suggesting that this is the origin of a compact outflow unresolved in their $14^{\prime \prime}$ resolution observations. However, from the lack of a bipolar distribution of the brightest CO-emitting gas in the inner regions of the $\mathrm{CO}$ cloud, they conclude that the source of the very extended outflow is now inactive and therefore hard to identify. 

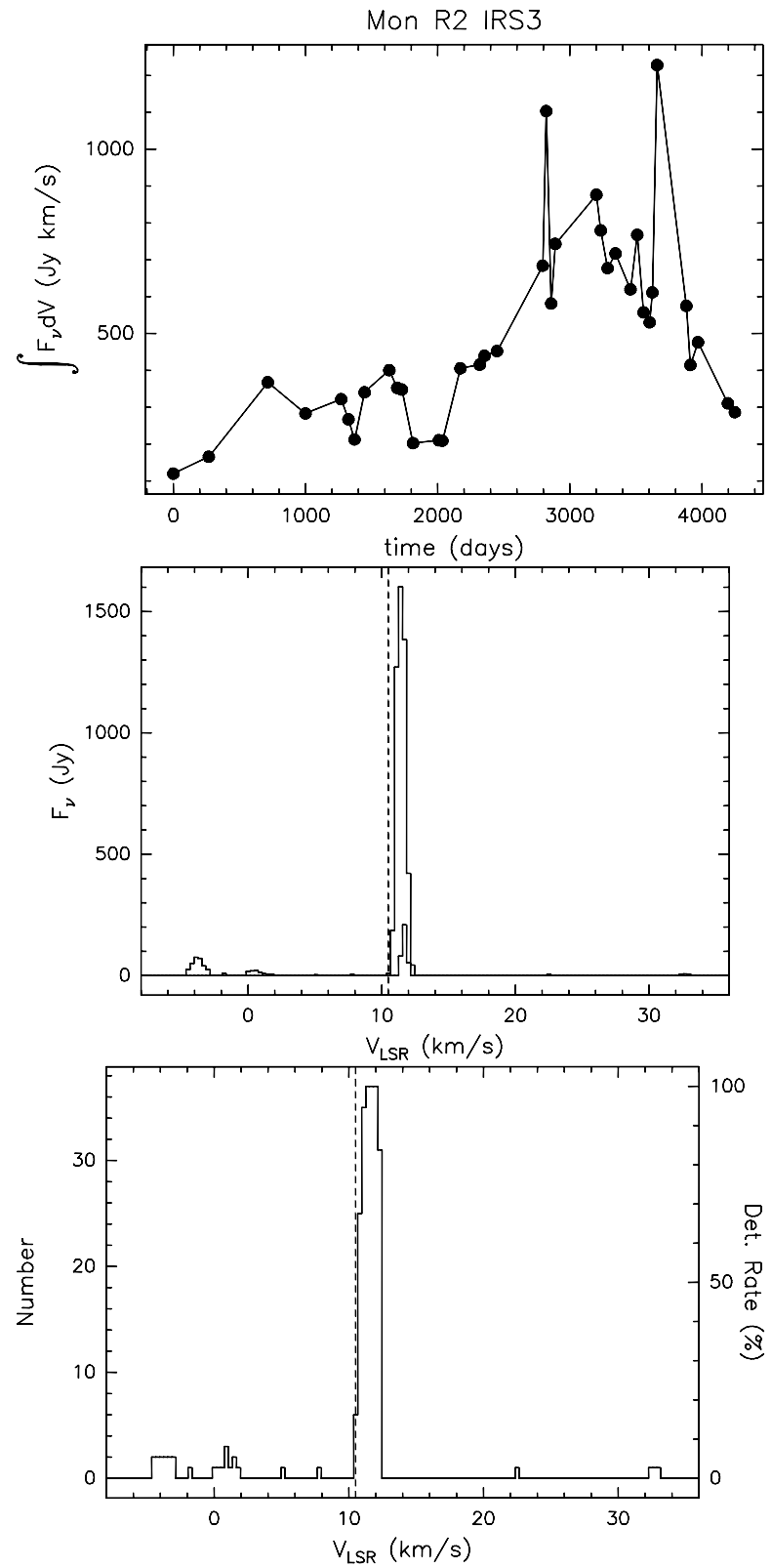

Fig. 10. Same as Fig. 4 for Mon R2 IRS3.

\subsubsection{Water maser properties}

The database consists of 38 spectra, extending over a period of 4250 days. From 1988 to 1990 we only have 1 spectrum per year. Starting in 1991, we have between 3 and 6 spectra per year, with an average time interval of 3.5 months. The maser is active at all times with a spectacularly constant velocity. Emission is confined in a single feature at $V_{\mathrm{LSR}}=11.5 \mathrm{~km} \mathrm{~s}^{-1}$ within a narrow velocity interval of $1.5 \mathrm{~km} \mathrm{~s}^{-1}$. This velocity is very close to that of the molecular cloud $V_{\mathrm{cl}}=10.5 \mathrm{~km} \mathrm{~s}^{-1}$ (Anglada et al. 1996). The presence of a single velocity feature makes Mon R2 and G32.74-0.08 (see below) unique among the surveyed sources. This is not due to the weakness of the maser (peak flux densities of 1500 Jy have been observed, as indicated in Fig. 10) and it is surprising given the large $\mathrm{CO}$ velocity widths observed at the same position.
On $12 / 05 / 1998$, a narrow feature appeared at $V_{\mathrm{LSR}}=$ $-3.7 \mathrm{~km} \mathrm{~s}^{-1}$, with a peak intensity of $75 \mathrm{Jy}$. In the next run seven months later, emission at this velocity is not detected. Finally, a weak line of $7 \mathrm{Jy}(3 \sigma)$ shows up in the last observation at $V_{\mathrm{LSR}}=+5.5 \mathrm{~km} \mathrm{~s}^{-1}$.

The integrated flux density (see Fig. 10) shows a variation of a factor of 10 over an interval of 10 years. The peak flux density remains always above $200 \mathrm{Jy}$, although fluxes as low as 8 Jy have been reported in the literature (Genzel \& Downes 1977).

\subsection{Sh 2-269 IRS2}

\subsubsection{The maser environment}

Sh 2-269 IRS2 is a small $\left(\sim 3^{\prime}\right)$ optically visible Hil region. A molecular cloud, probably located behind the nebula, has been detected in the same region (Wouterloot \& Brand 1989; Bronfman et al. 1996; Zinchenko et al. 1998). A dust lane running almost N-S bisects the nebula, towards the center of which there is an early-type star (B0.5V), responsible for most of the observed ionization.

The radio continuum emission shows a rather different picture since it is present only in the western lobe of the nebula and requires an exciting star of a much earlier spectral type, i.e. O8-O9.5 (Israel 1976; Fich 1993). The near-infrared source IRS2 is located at the peak of the radio continuum emission and is believed to correspond to the highly extincted YSO required for the ionization of the radio source (Persi et al. 1989; Pismis \& Mampaso 1991; Eiroa et al. 1994).

The $\mathrm{H}_{2} \mathrm{O}$ and $\mathrm{OH}$ masers are located very close to IRS2 and the radio peak. Large $\mathrm{CO}$ wings at the same position (Wouterloot \& Brand 1989) suggest that the IRS2 produces an outflow. The interaction of the flow with the ambient medium could give rise to the $\mathrm{HH}$ object (No. 191) detected in $\mathrm{H} \alpha$ and [SII] (Eiroa et al. 1994).

\subsubsection{Water maser properties}

The database consists of 51 spectra, extending over a period of 4659 days. At almost any time the spectrum presents three lines at velocities of approximately 16.2 , 19.5 and $20.5 \mathrm{~km} \mathrm{~s}^{-1}$, with the central one very close to $V_{\mathrm{cl}}$ (see Fig. 12). Such a simple structure of the spectrum is not limited to the period of time covered by our monitoring: even in the earliest observations of the maser found in the literature, the spectral profile is quite similar, with some significant difference only in the relative intensity of the lines (e.g. Genzel \& Downes 1977). Note the presence of a broad feature around $8 \mathrm{~km} \mathrm{~s}^{-1}$ toward the end of our monitoring, which was never found before and in earlier observations as reported in the literature.

The histogram of the frequency of occurrence shows three almost identical peaks at the above mentioned velocities. The variation of the intensity is less extreme than in the other sources of the sample. In particular, the line at $19 \mathrm{~km} \mathrm{~s}^{-1}$ seems quite stable in intensity during the 

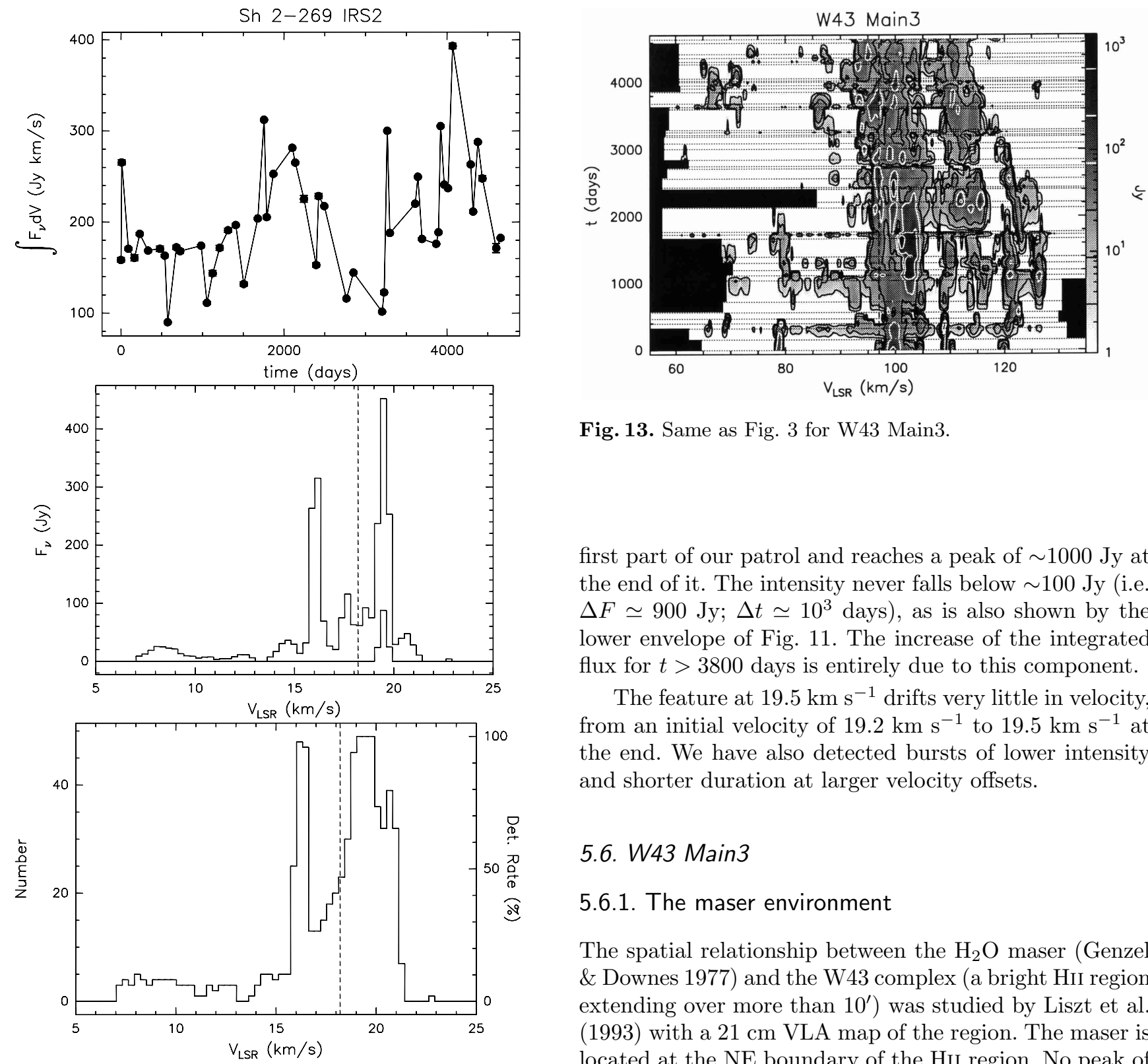

Fig. 13. Same as Fig. 3 for W43 Main3.

first part of our patrol and reaches a peak of $\sim 1000 \mathrm{Jy}$ at the end of it. The intensity never falls below $\sim 100 \mathrm{Jy}$ (i.e. $\Delta F \simeq 900 \mathrm{Jy} ; \Delta t \simeq 10^{3}$ days), as is also shown by the lower envelope of Fig. 11. The increase of the integrated flux for $t>3800$ days is entirely due to this component.

The feature at $19.5 \mathrm{~km} \mathrm{~s}^{-1}$ drifts very little in velocity, from an initial velocity of $19.2 \mathrm{~km} \mathrm{~s}^{-1}$ to $19.5 \mathrm{~km} \mathrm{~s}^{-1}$ at the end. We have also detected bursts of lower intensity and shorter duration at larger velocity offsets.

\subsection{W43 Main3}

\subsubsection{The maser environment}

The spatial relationship between the $\mathrm{H}_{2} \mathrm{O}$ maser (Genzel \& Downes 1977) and the W43 complex (a bright Hit region extending over more than $10^{\prime}$ ) was studied by Liszt et al. (1993) with a $21 \mathrm{~cm}$ VLA map of the region. The maser is located at the NE boundary of the HII region. No peak of continuum emission is present at the maser position with a resolution of 12 .' 5 . Maps of the region in the $\mathrm{HCO}^{+}$and ${ }^{13} \mathrm{CO}$ lines (Liszt 1995) show that at the position of the maser there is a distinct molecular peak in the extended and massive molecular cloud associated with W43. The peak is particularly evident in the $\mathrm{HCO}^{+}$channel maps between 95.5 and $100.5 \mathrm{~km} \mathrm{~s}^{-1}$.

The W 43 region has also been observed by Becker et al. (1994) in their $6 \mathrm{~cm}$ survey of the galactic plane with a resolution of $4^{\prime \prime}$. The radio component closest to the maser position is G30.785-0.030 $\left(\alpha_{1950}=18^{\mathrm{h}} 45^{\mathrm{m}} 01^{\mathrm{s}} 6\right.$, $\delta_{1950}=-01^{\circ} 58^{\prime} 49^{\prime \prime}$. 1$)$, with an integrated flux density greater than 850 mJy. According to Becker et al. (1994), this radio component is associated with the bright IRAS source IRAS 18449-0158 and classified as an UC HII region. The velocity of the molecular cloud is in good agreement with that of the maser. For our analysis, we associate this IRAS source with the $\mathrm{H}_{2} \mathrm{O}$ maser even though the separation is larger than the Medicina HPBW. 

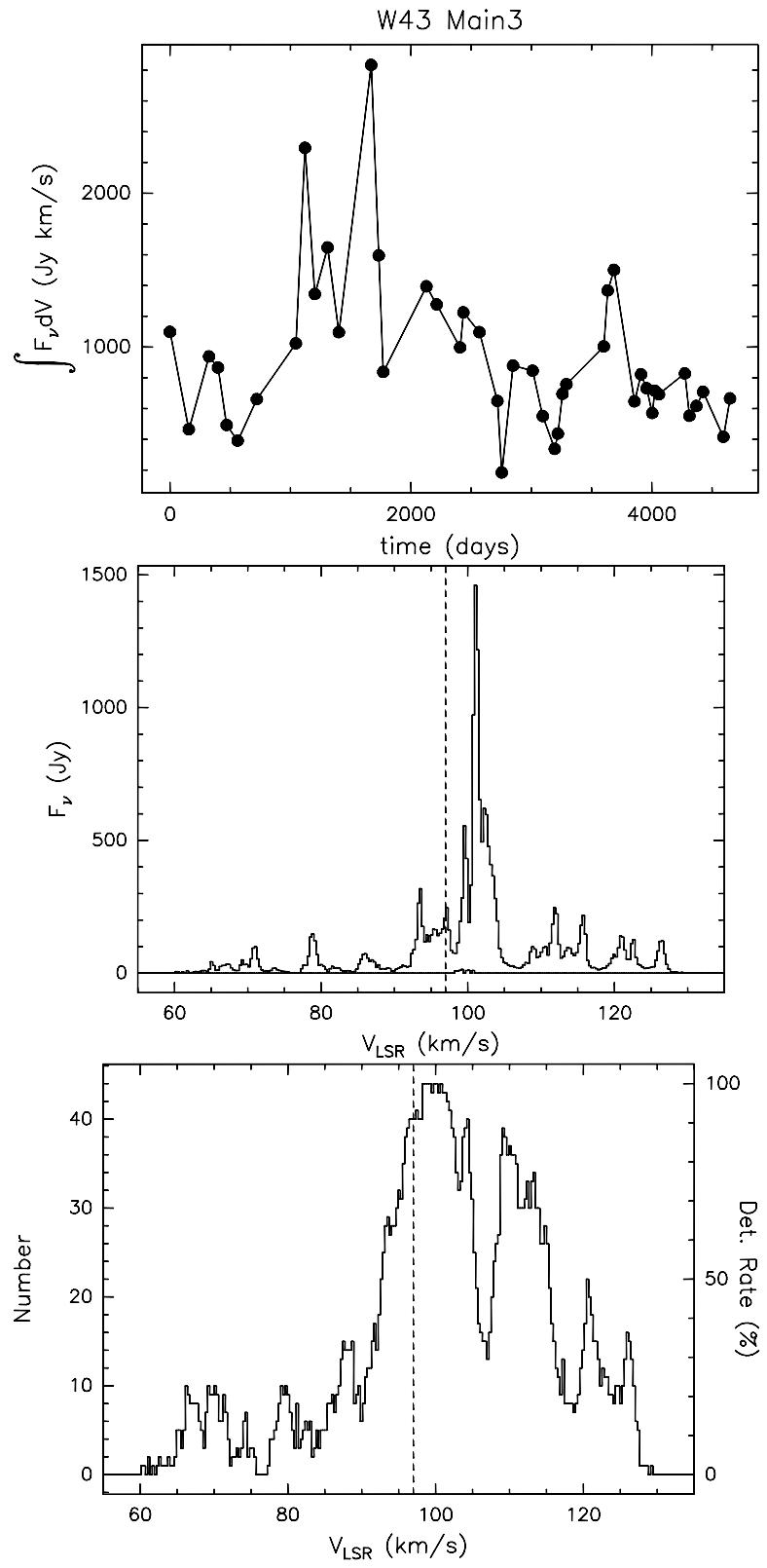

Fig. 14. Same as Fig. 4 for W43 Main3.

\subsubsection{Water maser properties}

This source has always shown many different velocity components. The first spectrum (taken in 1976) shows more than 15 features centred at $100 \mathrm{~km} \mathrm{~s}^{-1}$ (Genzel \& Downes 1977). In 1982, the spectrum exhibits a very complex structure, with more than 10 separate velocity components, and has undergone considerable changes since the first observations as there is little detailed velocity correspondence between the two spectra (Matthews et al. 1985).

The source has been observed regularly with no major gaps in time (apart from the period between 02/92 and $01 / 93)$. The average time interval between consecutive observations is 3.4 months. A total of 45 spectra have been obtained. As already indicated in the earlier observations, the emission shown in Fig. 13 presents a large number of lines in the velocity interval 60 to $130 \mathrm{~km} \mathrm{~s}^{-1}$, all of them strongly varying.

The brightest outburst occurs on $t=1670 \mathrm{~d}$ in which a component at $102 \mathrm{~km} \mathrm{~s}^{-1}$ reaches a peak flux density of $1500 \mathrm{Jy}$ and lasts for a period smaller than our sampling interval. As a consequence, the upper envelope of Fig. 14 is dominated by this outburst.

Many minor isolated bursts occur at large velocity offsets (red and blue), without any apparent time correlation and usually with a duration comparable with our sampling interval and a lower $\Delta F$ than those occurring near $V_{\mathrm{cl}}$. The only velocity component that can be traced for several consecutive observations is that at $99 \mathrm{~km} \mathrm{~s}^{-1}$, peaking at $t=2408 \mathrm{~d}$.

There is a general tendency of drifting in velocity (from higher to lower velocities), amounting to $\sim 2 \mathrm{~km} \mathrm{~s}^{-1}$ in 1000 days. This is particularly evident in the feature between 120 and $125 \mathrm{~km} \mathrm{~s}^{-1}$ and between days 1500 and 3000 .

Given the complexity of the source, maser emission could be the result of several independent components, all within the Medicina beam. Higher resolution observations are needed to separate the various components and to study their variability.

\section{7. $G 32.74-0.08$}

\subsubsection{The maser environment}

The positions of the $\mathrm{H}_{2} \mathrm{O}$ maser spots have been determined by Forster \& Caswell (1989) with an absolute accuracy of 0.5 . No HiI region associated with the maser has been detected at $22 \mathrm{GHz}$ by the same authors, down to a $3 \sigma$ level of 80 mJy. However, at $5 \mathrm{GHz}$ Becker et al. (1994) have found a continuum source of $7 \mathrm{mJy}$, unresolved in their $4^{\prime \prime}$ beam: the position of this source is virtually coincident (separation $<1^{\prime \prime}$ ) with the strongest water maser spot seen by Forster \& Caswell (1989).

\subsubsection{Water maser properties}

The database consists of 35 spectra, extending over a period of 4577 days. In 1987 and 1988 we only have one spectrum per year, while no data are available for 1990 . Starting in 1991, we have obtained between 2 and 5 spectra per year, with an average time interval of 3.3 months.

At most epochs, the spectrum consists of a single feature at $\sim 32.5 \mathrm{~km} \mathrm{~s}^{-1}$, with in some spectra a fainter line at $36.4 \mathrm{~km} \mathrm{~s}^{-1}$, close to the cloud velocity. This weak secondary component has a peak intensity of $16 \mathrm{Jy}$ and $\Delta t \simeq 18$ months. From Fig. 15 one sees that at some point the strongest feature has slightly shifted toward bluer velocities. These properties resemble those observed in Mon R2 IRS3. The integrated flux density variation shown in Fig. 16 basically reflects the variation of this component. Comparison between the upper and lower envelope (see Fig. 16) indicates a $\Delta F \simeq 80 \mathrm{Jy}$. 


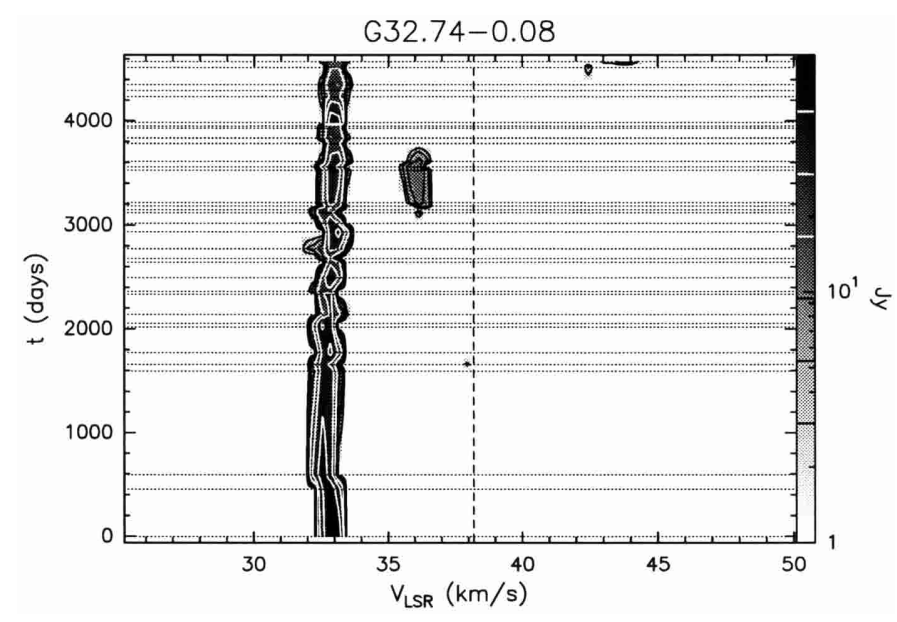

Fig. 15. Same as Fig. 3 for G32.74-0.08.

\section{8. $G 34.26+0.15$}

\subsubsection{The maser environment}

This is a well-known star forming complex in the W44 region, which has been extensively studied especially at radio wavelengths. Four HII regions are seen in the radio continuum in an area of $\sim 1^{\prime} \times 1^{\prime}$ (Gaume et al. 1994; Fey et al. 1994). Component $D$ is the more extended one $\left(\sim 1^{\prime}\right)$, has a ring-like shape, and is located on the eastern side of the complex. Component $\mathrm{C}$ is classified as a cometary $\mathrm{UC}$ HII region and is located on the western side of the complex. The other two components (A and B) are ultracompact and are believed to be ionized winds. They are located in front of the apex of the cometary nebula, in-between components D and C.

VLA observations indicate that the $\mathrm{H}_{2} \mathrm{O}$ maser emission arises in three distinct groups within a $50^{\prime \prime} \times 30^{\prime \prime}$ region (Foster \& Carswell 1989, 1999; Hofner \& Churchwell 1996; Fey et al. 1994). Most of the $\mathrm{H}_{2} \mathrm{O}$ spots are coincident with a molecular hot core lying in front of the apex of the cometary UC HII region. This hot core has been detected in various tracers (Heaton et al. 1989; Carral \& Welch 1992; Akeson \& Carlstrom 1996). The other two groups of maser spots lie at $\sim 25^{\prime \prime}$ and $\sim 45^{\prime \prime}$ from the hot core and do not have associated radio continuum emission. Their velocities do not differ significantly from those of the spots in the hot core.

The velocity range of the $\mathrm{H}_{2} \mathrm{O}$ masers goes from $-24.5 \mathrm{~km} \mathrm{~s}^{-1}$ to $120.8 \mathrm{~km} \mathrm{~s}^{-1}$ (Fey et al. 1994; Hofner \& Churchwell 1996) and is larger than that shown in Fig. 17. Usually, such a velocity spread in water masers is indicative of outflowing motions: indeed, in the case of those in the hot core a North-South velocity gradient has been detected both on a large scale $\left(\sim 40^{\prime \prime}\right)$ by Cesaroni et al. (1991) and inside the hot core by Akeson \& Carlstrom (1996). About 11 subgroups of $\mathrm{H}_{2} \mathrm{O}$ spots have been identified in the hot core. The other two groups are also split into several separate components. All of these components fall within the HPBW of Medicina.

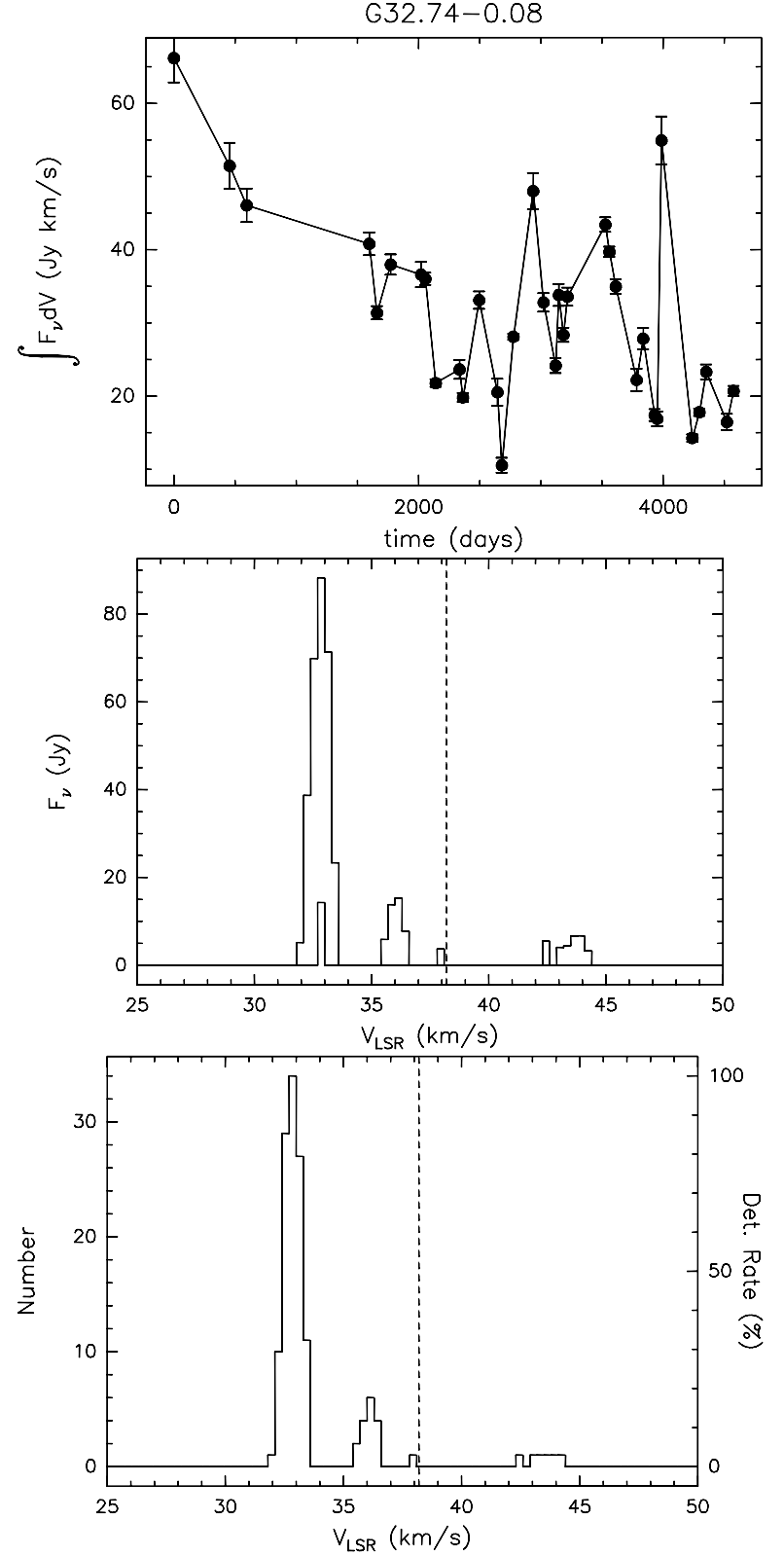

Fig. 16. Same as Fig. 4 for G32.74-0.08.

\subsubsection{Water maser properties}

A total of 46 spectra have been obtained, separated on average by 3.2 months. Unfortunately, it is very difficult to use our observations to study the $\mathrm{H}_{2} \mathrm{O}$ maser variability in this object: the low angular resolution of our single-dish observations mixes up the lines originating from the three different groups of spots and hence prevents us from disentangling any systematic variation which might originate in any of them.

There is a remarkably stable component at $V_{\mathrm{LSR}}=$ $60 \mathrm{~km} \mathrm{~s}^{-1}$, which is also the most intense of all the lines in this source. Comparison between the upper and lower envelope (see Fig. 18) indicates that this feature has a $\Delta F \lesssim 1800 \mathrm{Jy}$. The time variation of the integrated flux (see Fig. 18) also shows that the total emission is rather stable, with occasional bursts of short duration. 


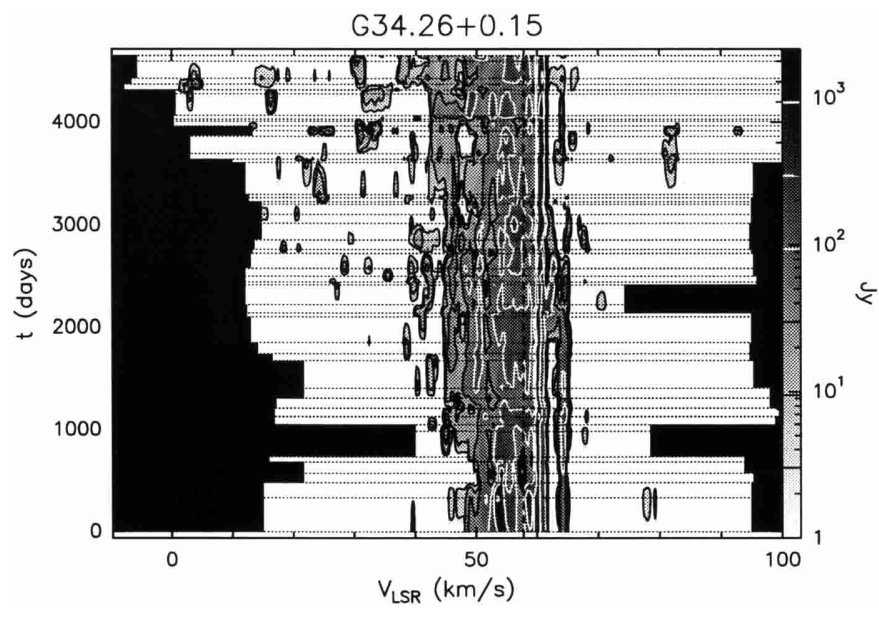

Fig. 17. Same as Fig. 3 for G34.26+0.15.

Most of the emission occurs between $V_{\mathrm{LSR}} \simeq 40$ and $65 \mathrm{~km} \mathrm{~s}^{-1}$, as indicated by the plot of the upper and lower envelope (Fig. 18) and of the frequency of occurrence (Fig. 18). Episodic bursts of low intensity and short duration occur at velocities $\leq 40 \mathrm{~km} \mathrm{~s}^{-1}$ and $\geq 65 \mathrm{~km} \mathrm{~s}^{-1}$.

\section{9. $G 35.20-0.74$}

\subsubsection{The maser environment}

G35.20-0.74 is located in a molecular cloud with a compact HII region at its peak (e.g., Little et al. 1983; Brown et al. 1982; Matthews et al. 1984). A molecular outflow, seen in $\mathrm{CO}$ and $\mathrm{HCO}^{+}$, originates from the Hir region and is oriented in the NE-SW direction (Matthews et al. 1984; Dent et al. 1985). On a smaller scale, near the HiI region, a VLA map in $\mathrm{NH}_{3}$ with $7^{\prime \prime}$ resolution (Little et al. 1985) and a map in CS with $15^{\prime \prime}$ resolution (Dent et al. 1985), show the presence of a molecular structure elongated in a direction almost perpendicular to the outflow, which is interpreted as a rotating disk, possibly collimating the outflow.

High-resolution near-infrared observations in $K$-band (Walter et al. 1990) indicate the presence of a strong absorption feature coincident with the cloud observed in $\mathrm{NH}_{3}$ and CS. Polarimetric observation in the $K$-band show an absorption lane which is presumably where the exciting star is located.

VLA continuum observations at $15 \mathrm{GHz}$ and $5 \mathrm{GHz}$ (Heaton \& Little 1988) resolve the HiI regions into three components aligned in the N-S direction, i.e. at about $45^{\circ}$ from the axis defined by the CO outflow. The central HII region is located at the centre of the $2.2 \mu \mathrm{m}$ absorption lane while the other two are associated with knots of $2.2 \mu \mathrm{m}$ emission. The central component coincides with the exciting star, while the northern and southern components could be ionized jets emanating from the central star (Heaton \& Little 1988). These aspects, as well as other observations in $\mathrm{C}^{18} \mathrm{O}$ and $\mathrm{CI}$, have led Heaton \& Little (1988) and Little et al. (1998) to propose the
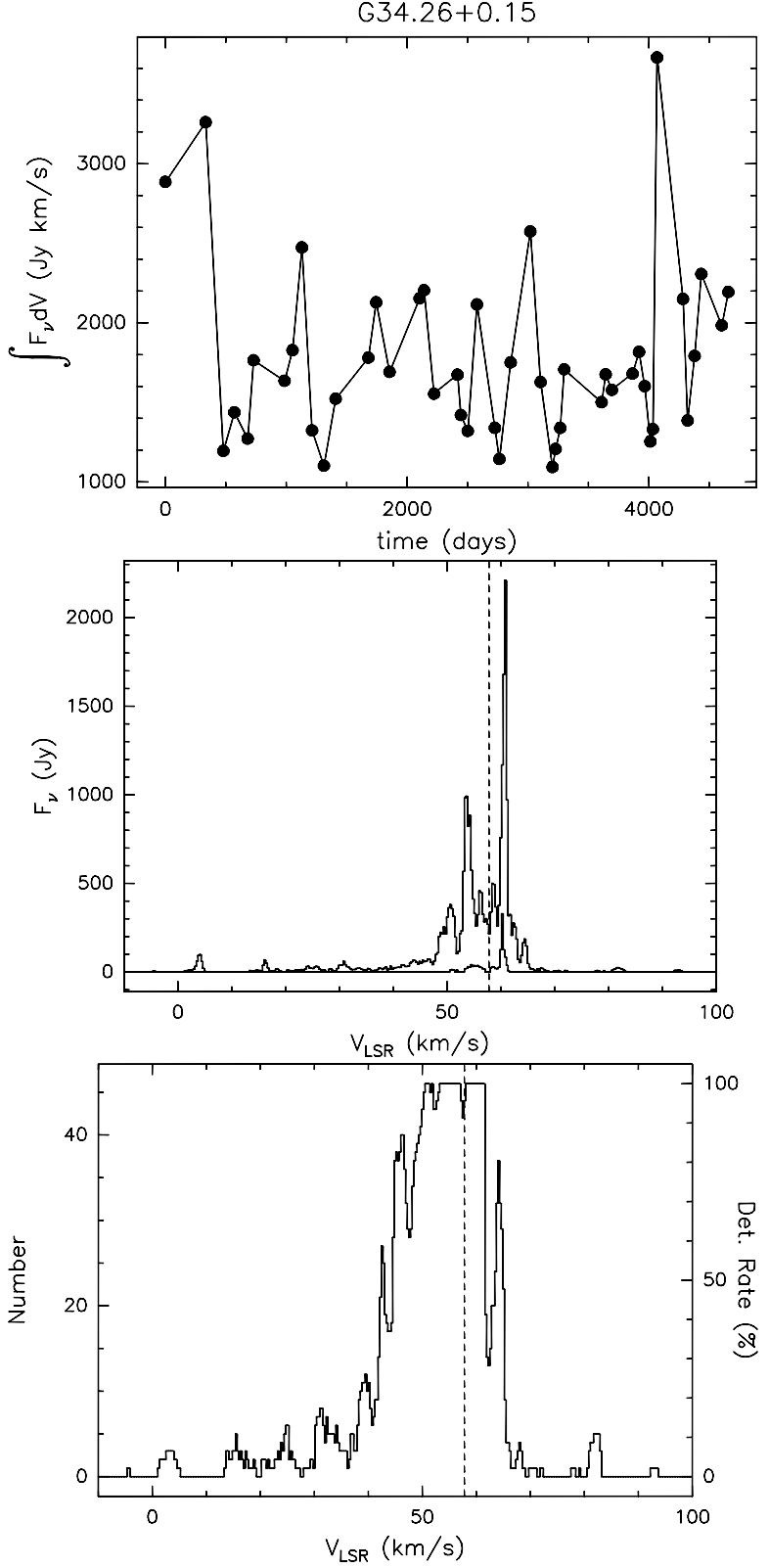

Fig. 18. Same as Fig. 4 for G34.26+0.15.

following model: a central star is surrounded by a molecular disk and emits jets in opposite directions which wander and precess to excavate a bipolar cavity and to drive a bipolar outflow.

The $\mathrm{OH}$ and $\mathrm{H}_{2} \mathrm{O}$ maser sources coincide with the central component of the HII region (Heaton \& Little 1988) and the position of the suggested driving source. High-resolution VLA observations of $\mathrm{OH}$ and $\mathrm{H}_{2} \mathrm{O}$ masers (Forster \& Caswell 1999) confirm that most of the maser components are in the same position as the central HiI region and are distributed in two sub-structures in the N-S direction with a maximum spatial separation of $5^{\prime \prime}$. 


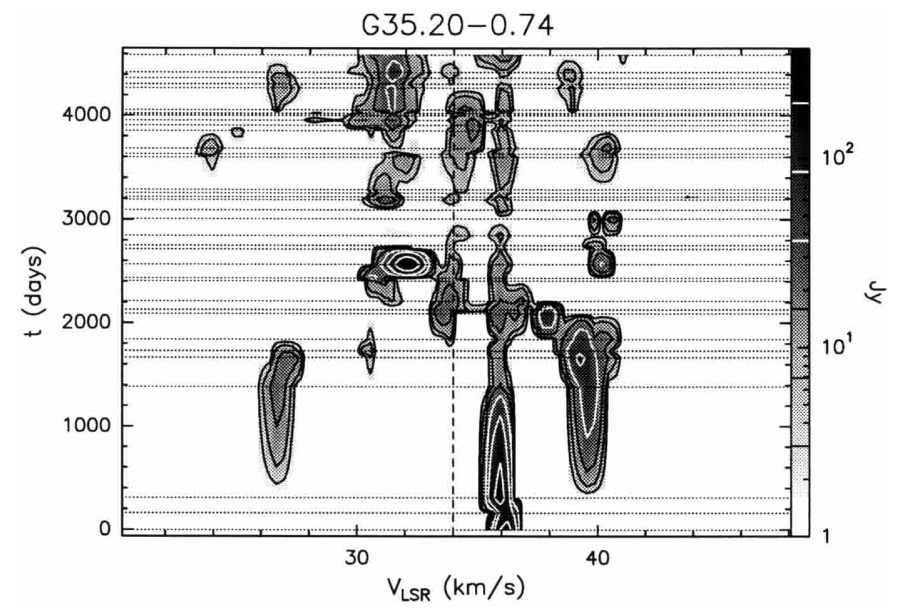

Fig. 19. Same as Fig. 3 for G35.20-0.74.

\subsubsection{Water maser properties}

The database consists of 36 spectra, extending over a period of 4594 days. In the first two years of observations (1987-88), we obtained only three spectra. Then, there has been a period of 35 months with no data points. Regular observations started again in 1991, with 2 to 5 spectra per year, and an average time interval between them of 3.4 months.

The maser is active at all times with a moderate flux density. Only one feature, at $V_{\mathrm{LSR}} \sim 36 \mathrm{~km} \mathrm{~s}^{-1}$, is present at almost all times. The variation of the integrated flux density shown in Fig. 20 presents only one major outburst due to a component at $V_{\mathrm{LSR}}=32 \mathrm{~km} \mathrm{~s}^{-1}$. As shown by the upper envelope in Fig. 20, emission extends from $\sim 22$ to $\sim 42 \mathrm{~km} \mathrm{~s}^{-1}$ with the strongest lines clustered around $V_{\mathrm{LSR}} \sim 35 \mathrm{~km} \mathrm{~s}^{-1}$.

In the majority of the events, the maser emission has a short duration, with $\Delta t$ ranging from less than our time resolution of $\simeq 3$ months to one year and $\Delta F$ up to $400 \mathrm{Jy}$. Components with large velocity offsets from $V_{\text {up }}$ tend to have smaller $\Delta t$ and $\Delta F$. These properties are illustrated in Fig. 20 which gives the frequency of occurrence of maser emission. The distribution is rather broad over the velocity interval $30-40 \mathrm{~km} \mathrm{~s}^{-1}$, without a main central peak. Velocity drifts can be seen in Fig. 19 with gradients of the order of $1 \mathrm{~km} \mathrm{~s}^{-1} \mathrm{yr}^{-1}$.

In 1984, according to VLA observations by Forster \& Caswell $(1989,1999) \mathrm{H}_{2} \mathrm{O}$ maser emission was concentrated mainly into three groups of components at $V_{\mathrm{LSR}}=$ $20 \mathrm{~km} \mathrm{~s}^{-1}, V_{\mathrm{LSR}}=31 \mathrm{~km} \mathrm{~s}^{-1}$ and $V_{\mathrm{LSR}}=37 \mathrm{~km} \mathrm{~s}^{-1}$. During the time interval of our observation the first of these groups remainse below our sensitivity. Only toward the end of the period (1997) a faint component arises at $V_{\mathrm{LSR}}=23.5 \mathrm{~km} \mathrm{~s}^{-1}$.

In the first part of the observing period, there is some anticorrelation between the feature at $V_{\mathrm{LSR}}=36 \mathrm{~km} \mathrm{~s}^{-1}$ and those at $V_{\mathrm{LSR}}=26.5 \mathrm{~km} \mathrm{~s}^{-1}$ and $V_{\mathrm{LSR}}=39 \mathrm{~km} \mathrm{~s}^{-1}$. It appears that the latter two features reach the maximum when the first one is at the minimum.

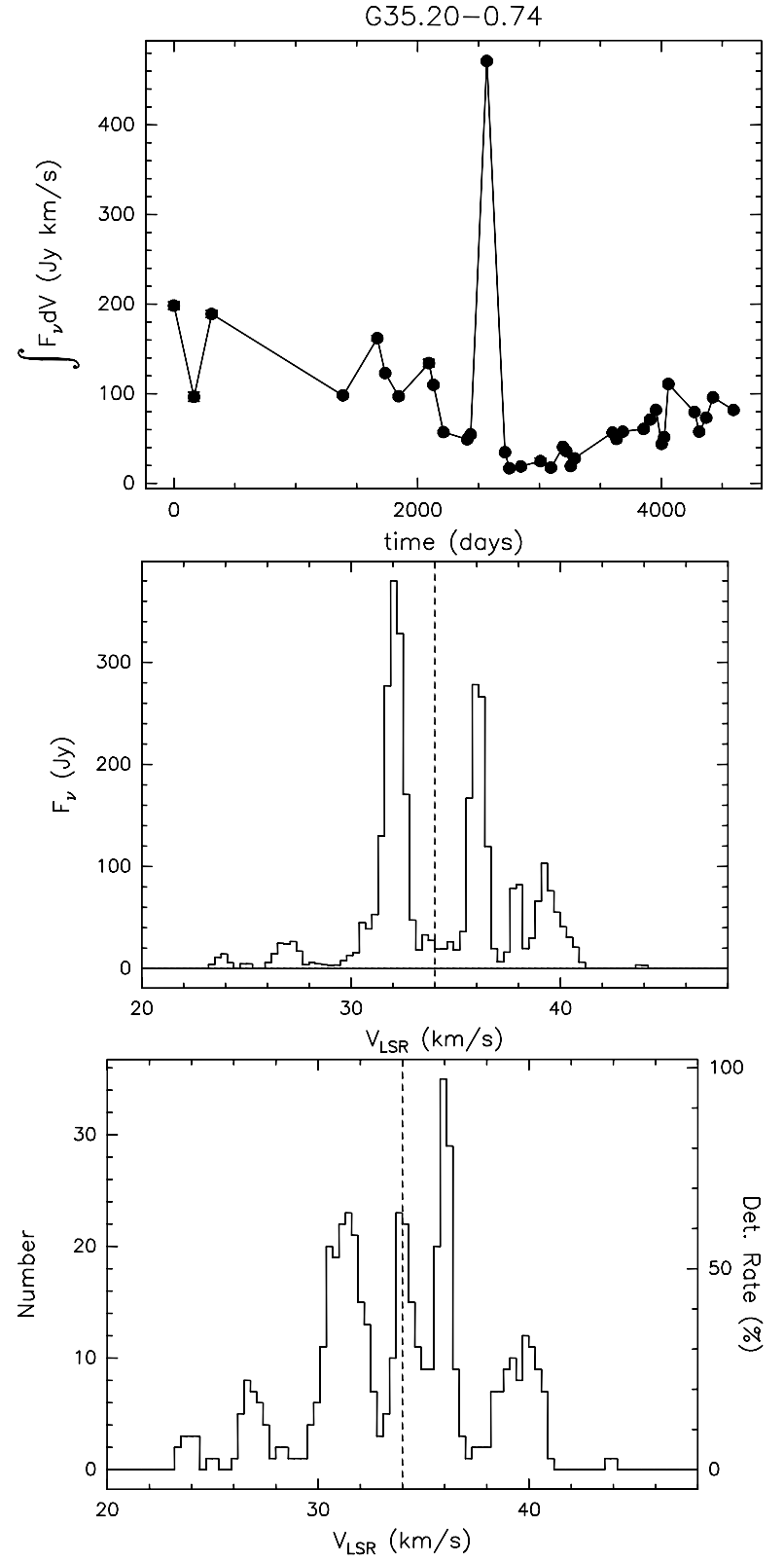

Fig. 20. Same as Fig. 4 for G35.20-0.74.

\subsection{0. $G 59.78+0.06$}

\subsubsection{The maser environment}

Molecular emission in the direction of the maser was detected in CS(7-6) by Plume et al. (1992) at a velocity of $22.3 \mathrm{~km} \mathrm{~s}^{-1}$ and in the $\operatorname{CS}(2-1), \operatorname{CS}(3-2)$ and $\operatorname{CS}(5-4)$ lines by Plume et al. (1997) at approximately the same velocity, confirming the presence of high density molecular gas at this position. Lada et al. (1981) made VLBI observations, and could derive very accurate coordinates for the maser $\left(\alpha_{1950}=19^{\mathrm{h}} 41^{\mathrm{m}} 04.27, \delta=23^{\circ} 36^{\prime} 42^{\prime \prime} \cdot 2\right)$. They identified three separate components all within one tenth of an arcsec. The most intense component (20 Jy) was at $12 \mathrm{~km} \mathrm{~s}^{-1}$, about $10 \mathrm{~km} \mathrm{~s}^{-1}$ blueshifted with respect to the molecular cloud velocity. The other two $(\leq 5) \mathrm{Jy}$ were at at 18.7 and $20.6 \mathrm{~km} \mathrm{~s}^{-1}$, closer to the CS velocity. 


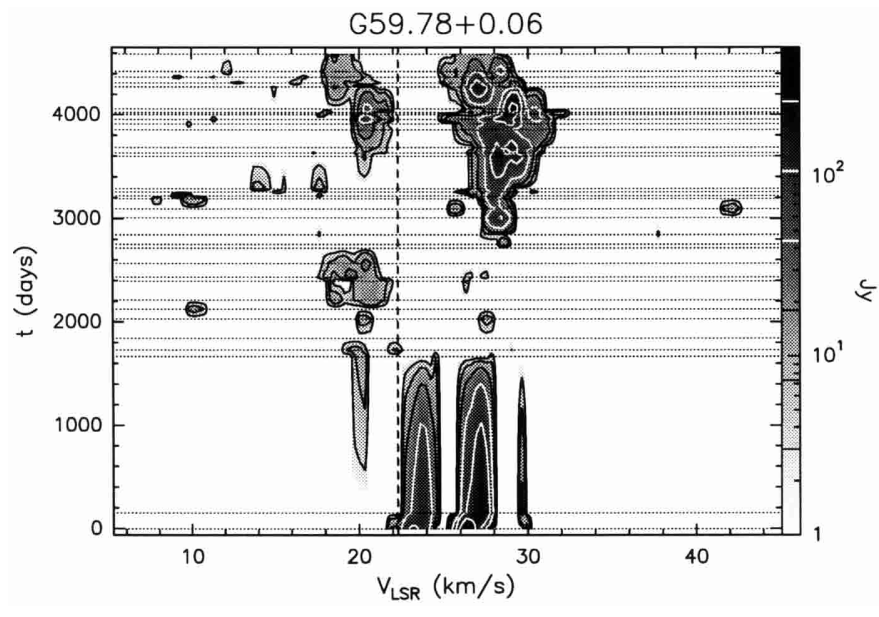

Fig. 21. Same as Fig. 3 for G59.78+0.06.

The maser is in the area of the very extended (more than $0.5^{\circ}$ in size) HII region S86, detected in lowresolution $21 \mathrm{~cm}$ radio continuum observations (Felli \& Churchwell 1972). Inspection of this low-resolution map, as well as of the $5 \mathrm{GHz}$ galactic plane survey of Altenhoff et al. (1978), does not show any prominent feature at the maser position. The absence of any small-diameter continuum source near the maser is confirmed in the Zoonematkermani et al. (1990) VLA $20 \mathrm{~cm}$ survey of the galactic plane, in which no source with flux density greater than $25 \mathrm{mJy}$ and size smaller than $20^{\prime \prime}$ is found at the position of the maser. Consequently, there might be no connection with the S86 nebula, or at least no radio continuum emission (within the above mentioned limits) seems to be directly associated with the maser. With the modern-day sensitivity a better upper limit (fraction of a mJy) should be put at higher frequencies to settle the issue. IRAS $19410+2336$ is within few arcseconds of the maser position and, in accordance with its colours, was classified as being of star-forming type by Palagi et al. (1993).

In summary, the maser seems to be associated with an intermediate-mass object (based on $L_{\mathrm{FIR}}$ ) in the earliest evolutionary phases, when an ionized region has not yet formed or it is so optically thick to be unobservable in the radio continuum. The protostar is also associated with a high-density molecular cloud.

\subsubsection{Water maser properties}

The time coverage is relatively uniform in the last part of the monitoring period, but presents a large gap of about 3 years after our first two observations. Consequently, in Fig. 21 the extension of the components after $t=200 \mathrm{~d}$ is an effect of the linear interpolation process.

According to Fig. 21, the $12 \mathrm{~km} \mathrm{~s}^{-1}$ component, which was the brightest in 1979 (Lada et al. 1981), is present only around $t=4400 \mathrm{~d}$. Sporadically and at low levels emission occurs at the velocity of the other two
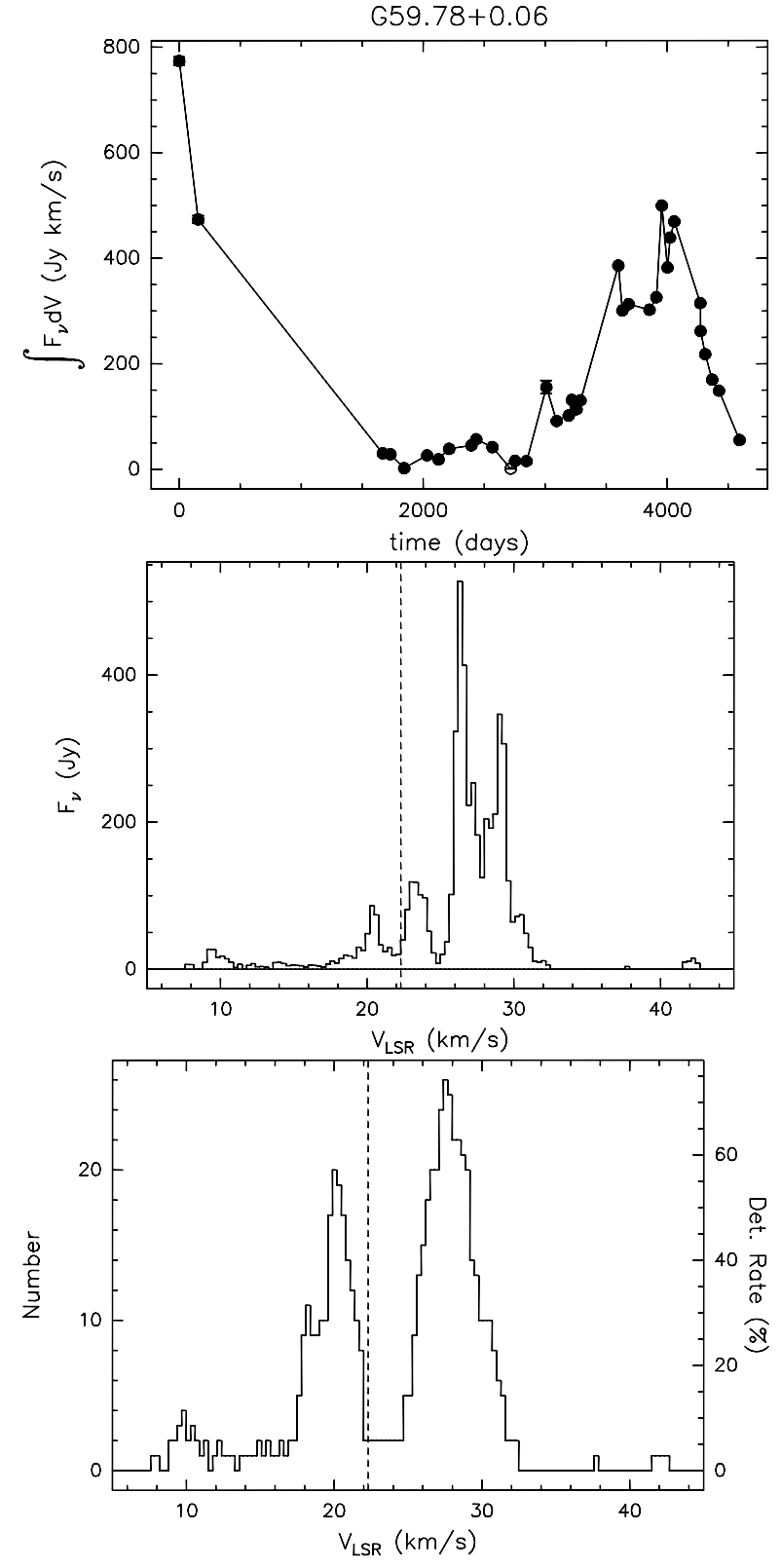

Fig. 22. Same as Fig. 4 for G59.78+0.06.

components detected by Lada et al. (1981), at 18.7 and $20.6 \mathrm{~km} \mathrm{~s}^{-1}$.

At two epochs $(t=1845 \mathrm{~d}$ and $t=2717 \mathrm{~d})$ the maser is totally quiescent, with no emission above the noise $(3 \sigma=$ $5 \mathrm{Jy})$ at any velocity.

The lifetime $\Delta t$ above the noise of a velocity component goes from a minimum of the order of a month (i.e. less than or equal to the minimum interval between two consecutive observations), to a maximum of the order of a few years. The upper and lower envelopes of the emission are shown in Fig. 22. As already noted, the lower envelope is zero, while the upper envelope shows emission mainly from 10 to $32 \mathrm{~km} \mathrm{~s}^{-1}$.

At the beginning of our patrol there are two main peaks at $\sim 23 \mathrm{~km} \mathrm{~s}^{-1}$ and $\sim 26 \mathrm{~km} \mathrm{~s}^{-1}$. In the two closely spaced observations these components show a 
velocity drift, corresponding to a velocity change of $<1 \mathrm{~km} \mathrm{~s}^{-1} \mathrm{yr}^{-1}$, of the same order as that found in other sources.

From $t=2847 \mathrm{~d}$ a new component at velocity $V_{\mathrm{LSR}} \sim$ $28 \mathrm{~km} \mathrm{~s}^{-1}$ emerges from the noise. This feature then splits into two peaks at different velocities. The velocity difference seems to be symmetrical relative to the initial velocity.

Emission at the extreme blue and red velocities occurs sporadically. In particular, one component appears at $V_{\mathrm{LSR}}=42 \mathrm{~km} \mathrm{~s}^{-1}$ on $t=3094 \mathrm{~d}$, then disappears, while a component appears a few months later at the other extreme, at $V_{\mathrm{LSR}}=10 \mathrm{~km} \mathrm{~s}^{-1}$.

\subsection{Sh $2-128\left(\mathrm{H}_{2} \mathrm{O}\right)$}

\subsubsection{The maser environment}

This $\mathrm{H}_{2} \mathrm{O}$ maser was first detected by $\mathrm{Ho}$ et al. (1981), and called $\mathrm{Sh} 2-128\left(\mathrm{H}_{2} \mathrm{O}\right)$ due to its proximity to the HiI region Sh 2-128. In fact, the maser is located $12^{\prime \prime}$ north of a compact $\left(<4^{\prime \prime}\right)$ HiI region (Sh 2-128 N), optically invisible, which is in turn located $60^{\prime \prime}$ north of the diffuse HiI region Sh 2-128, observed in the optical and in the radio continuum (Fich et al. 1986). In the 19791980 observations of Ho et al. (1981), the maser presents a main peak at $V_{\mathrm{LSR}}=-80 \mathrm{~km} \mathrm{~s}^{-1}$ (with peak intensity $\sim 100-150$ Jy over a six months period), and other lowerintensity features up to $V_{\mathrm{LSR}}=-70 \mathrm{~km} \mathrm{~s}^{-1}$. A more accurate position of the maser was obtained with the VLA by Hashick \& Ho (1985), who found two components, separated by $1 . .23$ in Right Ascension and 8.'6 in Declination (hence indistinguishable in our observations), and at velocities $V_{\mathrm{LSR}}=-76$ and $V_{\mathrm{LSR}}=-71 \mathrm{~km} \mathrm{~s}^{-1}$. No $\mathrm{H}_{2} \mathrm{O}$ masers have been found in other parts of the Sh 2-128 complex.

The Sh 2-128 N compact HII region and the two masers are at the centre of an elongated CO molecular cloud $(29 \times 9 \mathrm{pc}, 1985)$. The $\mathrm{CO}$ emission peaks at $V_{\mathrm{LSR}} \sim-73 \mathrm{~km} \mathrm{~s}^{-1}$ and the maser is located in a sharp transition zone where the velocity has a steep gradient, from $V_{\mathrm{LSR}}=-74 \mathrm{~km} \mathrm{~s}^{-1}$ west of the maser to $V_{\mathrm{LSR}}=-71.5 \mathrm{~km} \mathrm{~s}^{-1}$ east. $\mathrm{NH}_{3}$ is also detected at the position of the maser (1985). A possible interpretation of this configuration is that star formation is taking place at the interaction region of two colliding molecular clouds with different velocities. Recent $350 \mu \mathrm{m}$ images of massive star forming regions (Hunter et al. 2000) have detected thermal emission from heated dust around Sh 2-128 N and confirmed the presence of an embedded source.

\subsubsection{Water maser properties}

The database consists of 51 spectra, extending over a period of 4655 days and with an average time interval of 3.1 months. A detailed study of the maser variability over the period 1981-1994 is reported by Berulis et al. (1995). Their observations are in very good agreement

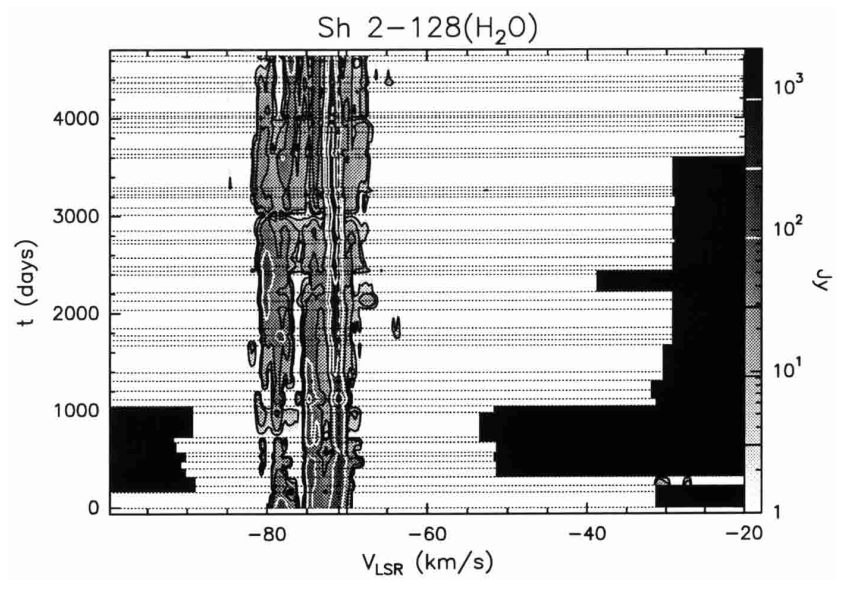

Fig. 23. Same as Fig. 3 for Sh $2-128\left(\mathrm{H}_{2} \mathrm{O}\right)$.

with ours in the overlapping periods. Berulis et al. (1995) distinguish between the emission in two velocity intervals, greater and lower than $V_{\mathrm{LSR}}=-75 \mathrm{~km} \mathrm{~s}^{-1}$. The emission at lower velocities shows the strongest burst in 1985, while that at $V_{\mathrm{LSR}}<-75 \mathrm{~km} \mathrm{~s}^{-1}$ begins to increase toward the end (1992-94) of their patrol. The emission at $V_{\mathrm{LSR}}=-75 \mathrm{~km} \mathrm{~s}^{-1}$ is generally weaker.

Figure 23 shows that during the period of our fairly uniform coverage the emission is dominated by a component at $V_{\mathrm{LSR}}=-72 \mathrm{~km} \mathrm{~s}^{-1}$ while the components at $V_{\mathrm{LSR}}>-72 \mathrm{~km} \mathrm{~s}^{-1}$ are visible only during a fraction of our patrol and at a lower intensity. The component at $V_{\mathrm{LSR}}=-72 \mathrm{~km} \mathrm{~s}^{-1}$ is undergoing a steady increase in intensity (from $\sim 200 \mathrm{Jy}$ at the beginning to a peak of $2000 \mathrm{Jy}$ ), and also a minor drift in velocity of $\sim 1 \mathrm{~km} \mathrm{~s}^{-1}$ over $\sim 3$ years.

In Fig. 24 the upper and lower envelopes of the emission confirm that the stronger activity occurs at $V_{\mathrm{LSR}}<$ $-72 \mathrm{~km} \mathrm{~s}^{-1}$. In the lower envelope histogram weak emission is present at $V_{\mathrm{LSR}}=-71 \mathrm{~km} \mathrm{~s}^{-1}$. This behavior is similar to that observed by Berulis et al. (1995). Figure 24 also shows that none of the other velocity components is permanently active but that, at one time or another, all undergo a phase of quiescence.

The integrated flux indicates that variability occurs on long time scales, with a minimum at the beginning of our patrol, a maximum after $\sim 3000$ days, and a subsequent decline. Superimposed on this gradual variation, only few isolated bursts of small intensity and duration occur, much less frequently than in other masers of our sample.

The frequency of occurrence shown in Fig. 24 shows a minimum at $V_{\mathrm{LSR}} \approx 76 \mathrm{~km} \mathrm{~s}^{-1}$ as also found by Berulis et al. (1995), while it is rather flat over the two velocity intervals -81 to $-77 \mathrm{~km} \mathrm{~s}^{-1}$ and -75 to $-70 \mathrm{~km} \mathrm{~s}^{-1}$.

\subsection{NGC 7129/FIRS2}

\subsubsection{The maser environment}

The maser is located in the NGC 7129 region, a reflection nebula illuminated by three brigth stars, BD+651637, 

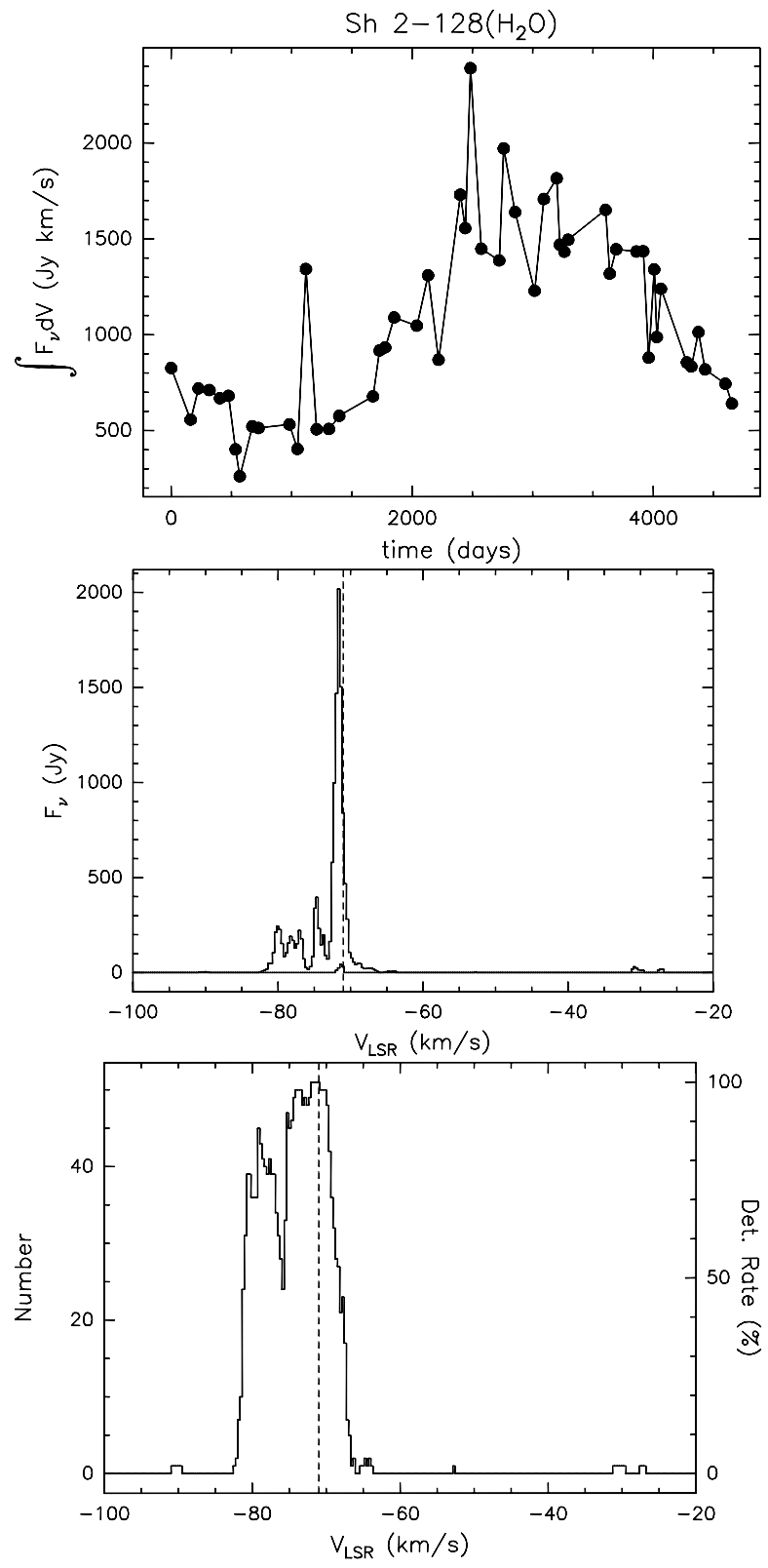

Fig. 24. Same as Fig. 4 for Sh $2-128\left(\mathrm{H}_{2} \mathrm{O}\right)$.

$\mathrm{BD}+651638$, and $\mathrm{LkH} \alpha 234$ (Harvey et al. 1984). There are two FIR sources in the region: FIRS1 associated with $\mathrm{LkH} \alpha 234$, and FIRS2 separated by $213^{\prime \prime}$ to the southwest (Bechis et al. 1978). High-resolution interferometric maps show that FIRS1 is coincident with the peak of a dense molecular core, whereas FIRS2 is located in a minimum of molecular emission (Fuente et al. 2000). However, both objects are detected as compact, strong continuum sources at $1.3 \mathrm{~mm}$. In particular, FIRS2 reveals the presence of two components (MM1 and MM2) separated by only $\sim 1^{\prime \prime}$ (see Fuente et al. 2000). The total luminosity of FIRS2 has been estimated to be about $430 L_{\odot}$ (Eiroa et al. 1998).

The major centre of activity, including strong bipolar outflows, jets and $\mathrm{H}_{2} \mathrm{O}$ maser emission is associated with $\operatorname{LkH} \alpha$ 234/FIRS1 (e.g. Ray et al. 1990). However,

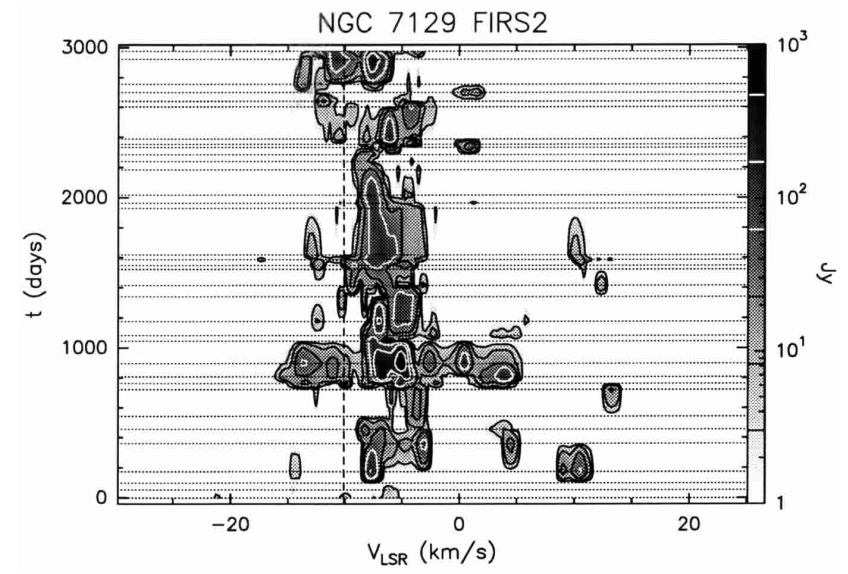

Fig. 25. Same as Fig. 3 for NGC 7129/FIRS2.

similar properties have been discovered recently around FIRS2: an $\mathrm{H}_{2}$ jet (Eislöffel 2000), and a high-velocity CO outflow (Fuente et al. 2000). Prior to our observations of April 1988 (in Comoretto et al. 1990 the maser is called $\mathrm{HH} 32-35), \mathrm{H}_{2} \mathrm{O}$ maser emission at the position of FIRS2 has been reported by Cesarsky et al. (1978) (called NGC 7129(1)). Their published spectrum showed two components at $V_{\mathrm{LSR}}=28$ and $38 \mathrm{~km} \mathrm{~s}^{-1}$ with respective flux densities of 12 and 5 Jy. Later detections (Rodríiguez et al. 1980; Sandell \& Olofsson 1981) have indicated that emission is coming from gas at $V_{\mathrm{LSR}}=-5$ to $-20 \mathrm{~km} \mathrm{~s}^{-1}$.

\subsubsection{Water maser properties}

The monitoring program for this maser began only in October 1991 and consists of 35 spectra. Prior to this date, we only have one observation made in April 1988 (not included in Fig. 25). From 1991 to 1999 we have made between 2 and 5 observing sessions per year, with an average time interval of 2.9 months.

The maser is active at all times with emission extending between $V_{\mathrm{LSR}} \sim-20$ and $\sim+15 \mathrm{~km} \mathrm{~s}^{-1}$. However, the main features arise at velocities around $-5 \mathrm{~km} \mathrm{~s}^{-1}$. A steady component is not present, and the maser emission appears to come from individual events that last at most for $\Delta t=1$ year and that are scattered over a large velocity interval.

The flux density is rather low (typically below $\sim 200 \mathrm{Jy}$ ), with the exception of a flare-like event that occurred in April 1994 with $\Delta t \sim 1$ yr and $\Delta F \simeq 1000 \mathrm{Jy}$.

\subsection{3. $L 1204-A$}

\subsubsection{The maser environment}

The L1204 molecular cloud contains several components in which star formation may be present (Tafalla et al. 1993). The most prominent core, called L1204-A, is associated with Sh 2-140, a well known high-mass star forming region. Sh 2-140 hosts a group of 3 infrared sources (IRS1, IRS2 

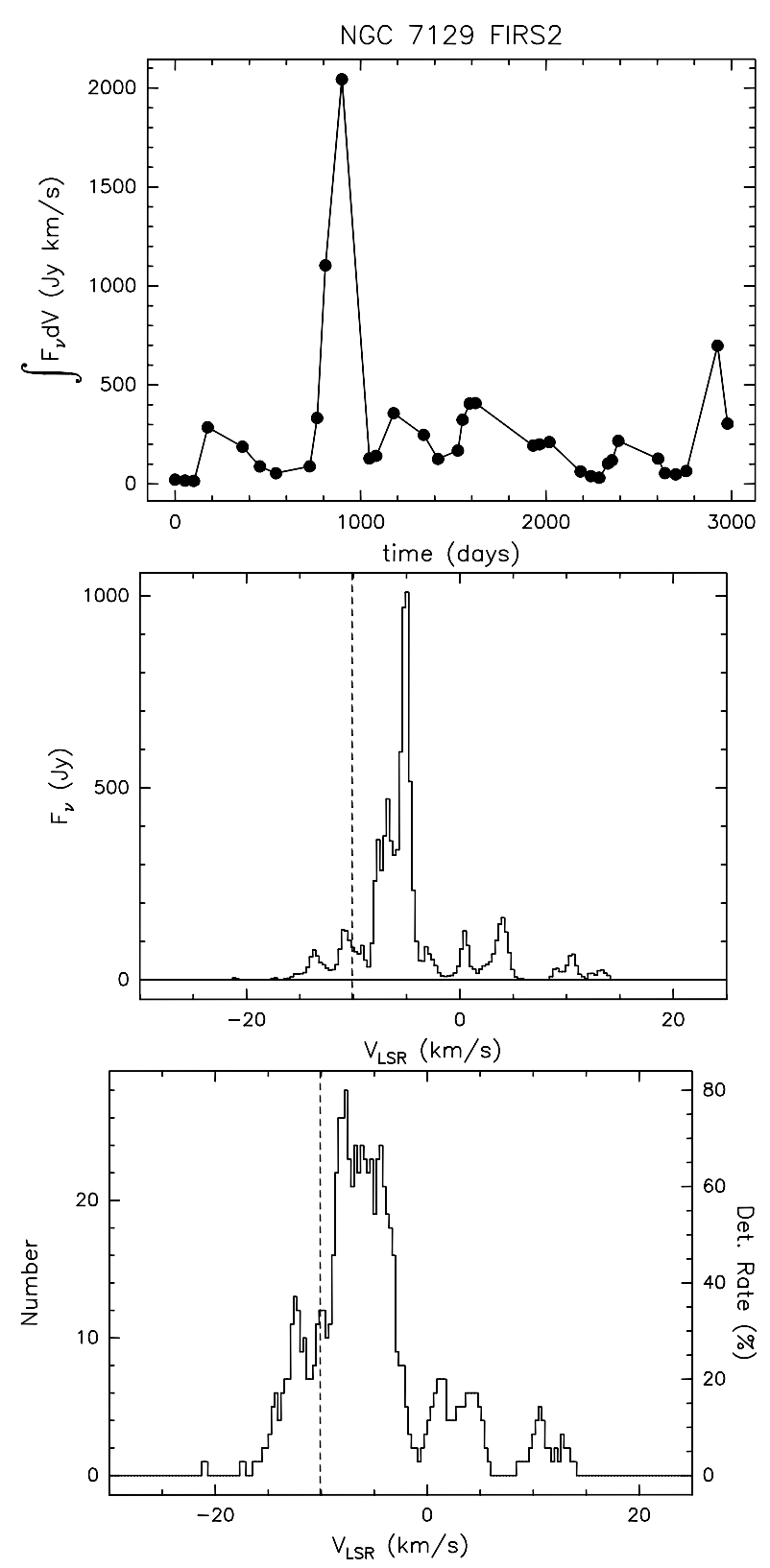

Fig. 26. Same as Fig. 4 for NGC 7129/FIRS2.

and IRS3), located on one side of a photodissociation region produced by the star HD 211880. VLA observations by Tofani et al. (1995) show that the $\mathrm{H}_{2} \mathrm{O}$ maser emission is associated with IRS1 and with a resolved HII region. The maser has two velocity components at $V_{\mathrm{LSR}}=-15 \mathrm{~km} \mathrm{~s}^{-1}$ and $V_{\mathrm{LSR}}=+4.1 \mathrm{~km} \mathrm{~s}^{-1}$.

L1204-A is the driving source of a molecular outflow whose red- and blue-shifted lobes are separated by $35^{\prime \prime}$ and have a high degree of overlap. The blueshifted lobe shows a faint extension toward the northern direction which almost completely overlaps the redshifted lobe. This morphology was interpreted as an almost pole-on outflow by many authors, see e.g. Hayashy et al. (1987), Phillips et al. (1988), and Minchin et al. (1993).

Large-scale observations in $\mathrm{NH}_{3}$ (Zhou et al. 1993) and CS (Hayashi \& Murata 1992) show a well-defined ring

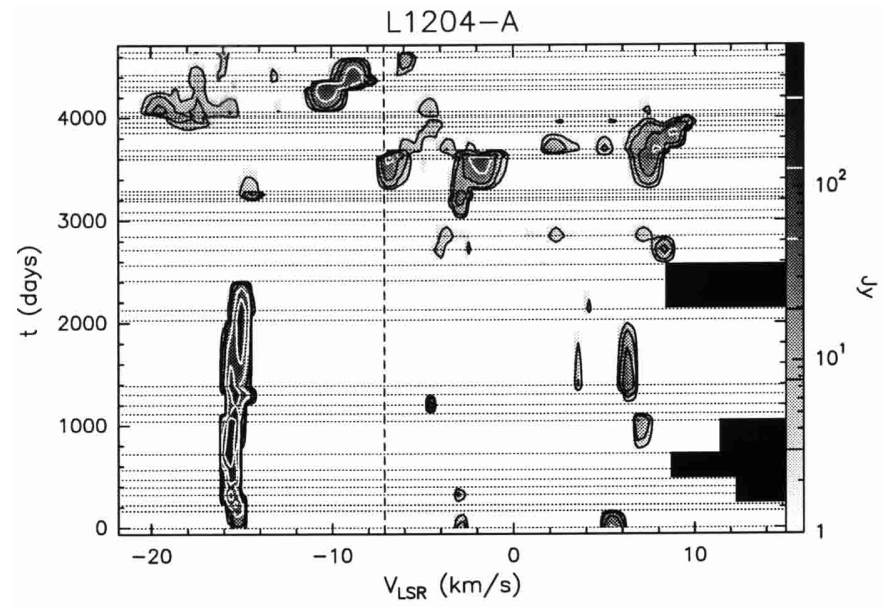

Fig. 27. Same as Fig. 3 for L1204-A.

which bounds the IR cluster in the South, while the northern part is missing.

Observations at $5^{\prime \prime}$ resolution of $\mathrm{HCO}^{+}$(Wilner \& Welch 1994) indicate the presence of a disk around IRS1 viewed almost edge-on with an inclination angle of $\leq 20^{\circ}$; moreover the same observations show that the lobes of the denser parts of the outflow are overlapped less than the outflow traced by CO.

Based on observations of the IR reflection nebula located around the IR cluster, Harker et al. (1997) suggest that IRS1 can be modelled as a protostar surrounded by an almost edge-on dusty disk. The large-scale structure reflects the interaction between the outflow and the surrounding material, but has no direct link with the geometry near the protostar.

\subsubsection{Water maser properties}

Lekht et al. (1993) have studied the time behaviour of this source over an interval of 11 years (1981-1992), which overlaps our period of observations by 6 years. Both sets of observations generally agree. Discrepancies in intensity can be attributed to calibration, while we may have lost some temporal feature due to less frequent observations.

Our observations extend those of Lekht et al. (1993) by about 7 years. After the big flare of the $V_{\mathrm{LSR}}=$ $-15.5 \mathrm{~km} \mathrm{~s}^{-1}$ component (Fig. 27), and the fading occurring in 1993, the maser source remains essentially quiet $\left(F_{\text {peak }} \leq 10 \mathrm{Jy}\right)$ for about two years. Apart from the $V_{\mathrm{LSR}}=-15.5 \mathrm{~km} \mathrm{~s}^{-1}$ component, which lasts for several years, the maser emission is composed of short duration ( $\Delta t<1$ year), small intensity bursts, dispersed over a large velocity range, from $V_{\mathrm{LSR}}=-20 \mathrm{~km} \mathrm{~s}^{-1}$ to $V_{\mathrm{LSR}}=+10 \mathrm{~km} \mathrm{~s}^{-1}$. This effect is illustrated in Fig. 28 where the upper envelope and frequency of occurrence show several peaks distributed over a large velocity range.

No clear evidence is found for the presence of three symmetric velocity components or for the anticorrelation between the central and side components found by Lekht et al. (1993). These facts, along with the VLA 

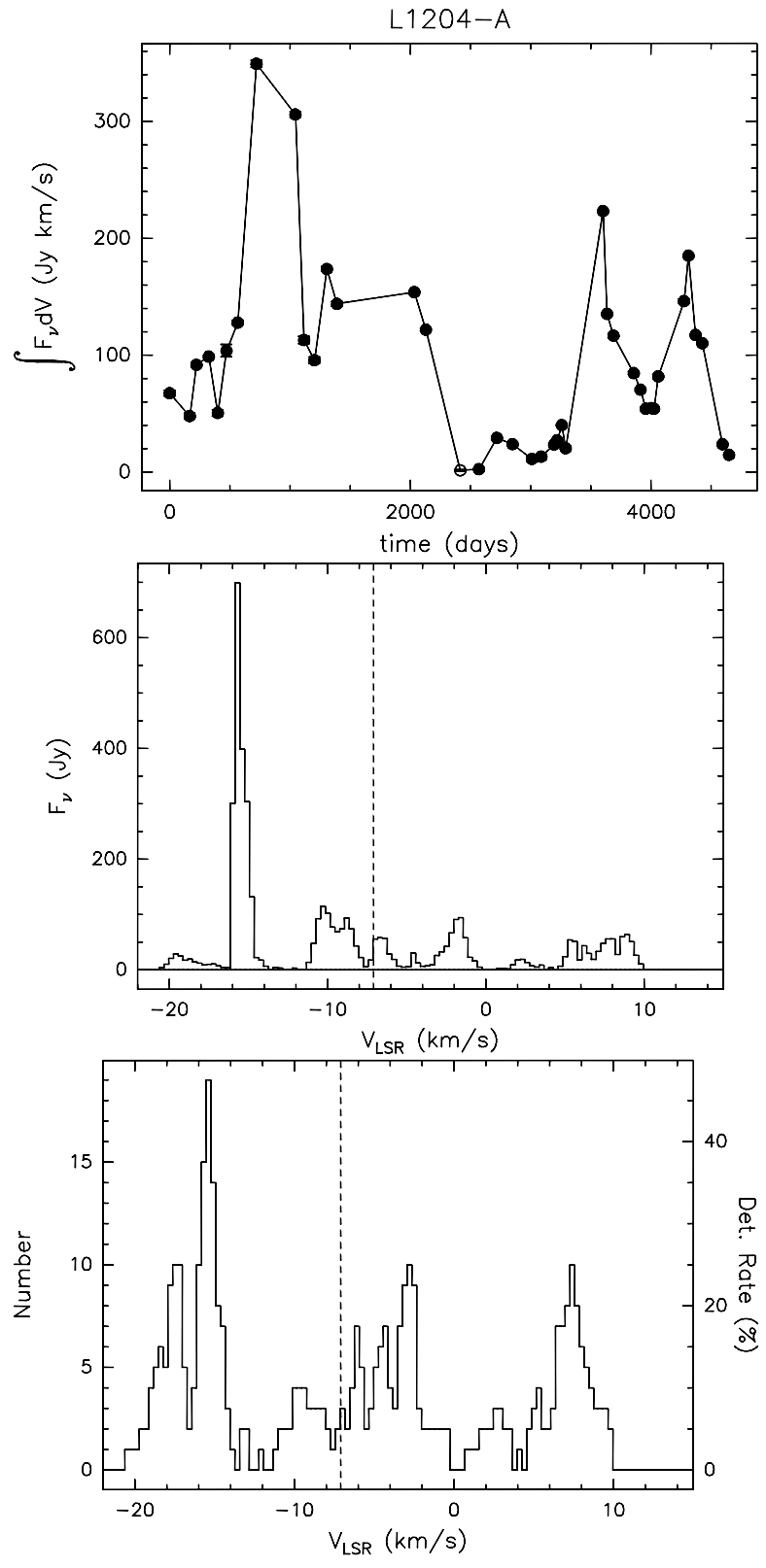

Fig. 28. Same as Fig. 4 for L1204-A.

observations of Tofani et al. (1995) which locate the $V_{\mathrm{LSR}}=-15 \mathrm{~km} \mathrm{~s}^{-1}$ component almost at the end of the jet and aligned with the so-called bullet detected by Schwartz (1989), do not confirm the interpretation of Lekht et al. (1993) that $\mathrm{H}_{2} \mathrm{O}$ masers follow the motion of a keplerian disk around the exciting source.

\subsection{4. $L 1204-G$}

\subsubsection{The maser environment}

L1204-G is located in the Sh 2-140 complex, at an offset of $\left(\Delta \alpha=34^{\prime}, \Delta \delta=14^{\prime}\right)$ from L1204-A. Molecularline maps in $\mathrm{NH}_{3}$ and $\mathrm{CS}$ have revealed the presence of a dense core at a systemic velocity of $\sim 11 \mathrm{~km} \mathrm{~s}^{-1}$ (Tafalla et al. 1993). Evidence for ongoing star formation is

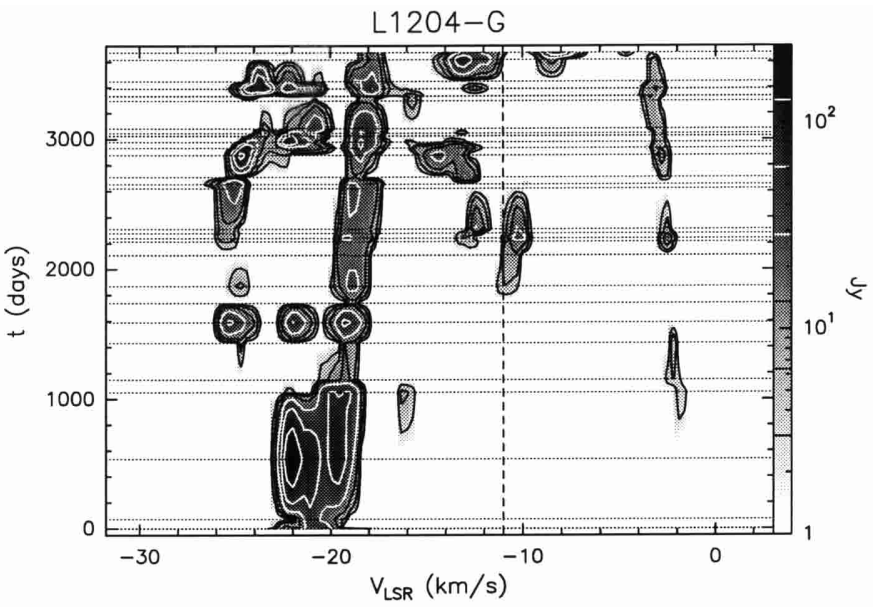

Fig. 29. Same as Fig. 3 for L1204-G.

deduced from the presence of an embedded IRAS source (IRAS 22198+6336), high-velocity CO gas, (Fukui 1989) and water maser emission (Palla et al. 1991). Both the luminosity of the IRAS source and the mass of dense gas are lower than in the case of L1204-A. However, L1204-G is the most prominent source besides L1204-A, within a region that contains four other molecular cores and several IRAS sources. Thus, it offers a good opportunity for a comparative study of the maser emission properties with L1204-A, and for this reason we have included it in our monitoring program. The large distance from L1204-A assures that our observations of L1204-G are not affected by the contribution of the brighter source.

\subsubsection{Water maser properties}

We have obtained 29 spectra over an interval of 11 years, with an average separation of 4.1 months. Initially, the coverage has been sparse, whereas regular observations of the source have been carried during the last 5 years of our patrol. The velocity-time-flux density contour plot of Fig. 29 clearly indicates that maser emission has been present at all times, with a major steady component at $V_{\mathrm{LSR}} \sim-20 \mathrm{~km} \mathrm{~s}^{-1}$. Interestingly, there is very little emission at the cloud systemic velocity. Like L1204-A, the emission extends over a velocity interval of about $30 \mathrm{~km} \mathrm{~s}^{-1}$ and shows several bursts of variable duration ( $\Delta t \simeq 200-500$ days, see Fig. 30 ). Despite the lower luminosity of the exciting IRAS source, the total integrated flux of L1204-G is approximately the same as that of L1204-A, as indicated in Table 2. Both the upper and lower envelopes (Fig. 30) and the frequency of distribution (Fig. 30) share the same characteristics as observed in L1204-A.

A global velocity drift of the main emission component toward the red is evident in Fig. 29. The magnitude of the velocity change is $\sim 0.2 \mathrm{~km} \mathrm{~s}^{-1} \mathrm{yr}^{-1}$. A similar, but opposite velocity drift of the feature at $V_{\mathrm{LSR}} \sim-2 \mathrm{~km} \mathrm{~s}^{-1}$ 

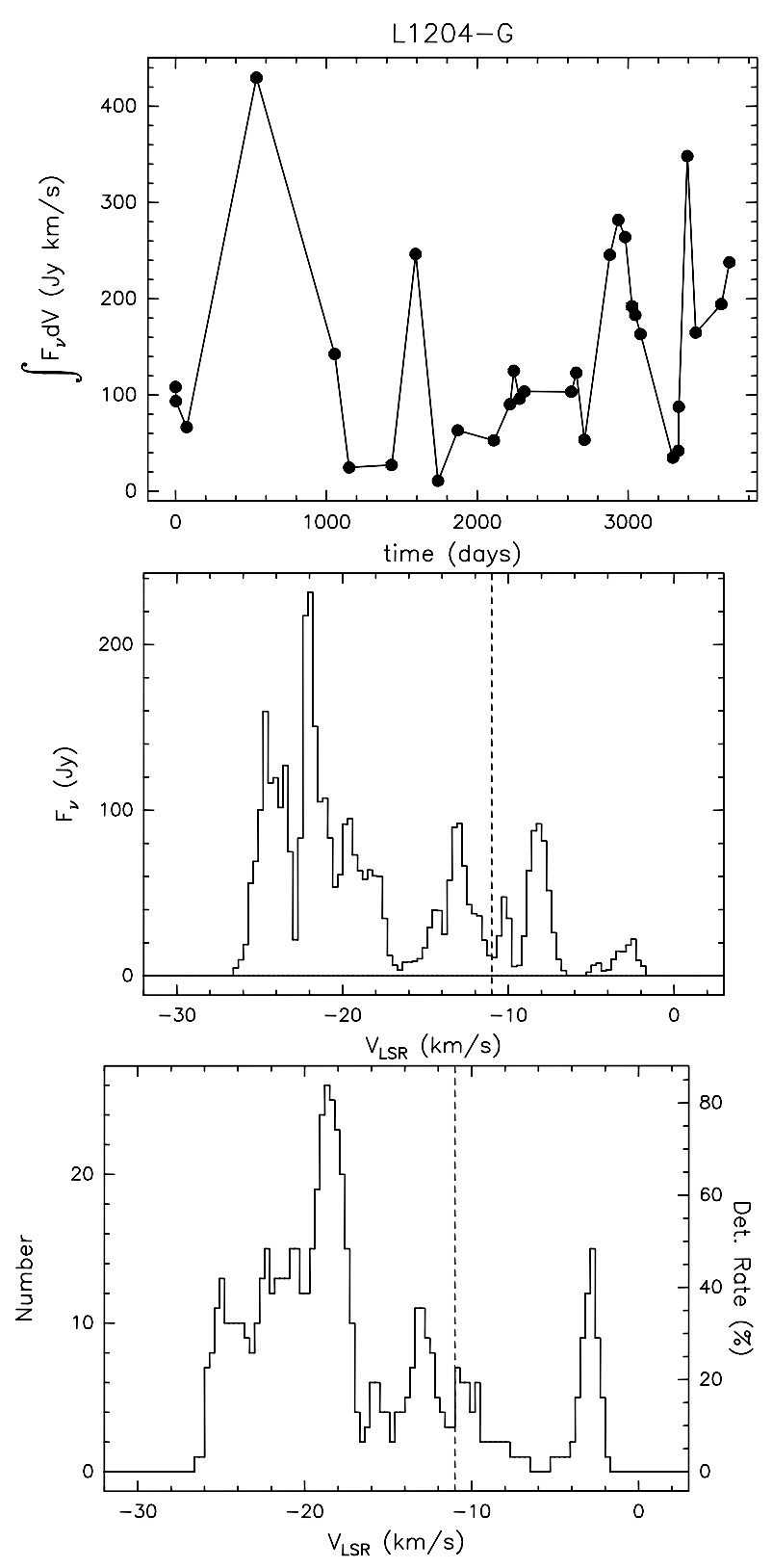

Fig. 30. Same as Fig. 4 for L1204-G.

is visible in the figure, although emission at this velocity is more sporadic.

\section{Conclusions}

We have presented the observational results of a systematic study of the water maser variability in 14 star-forming regions with luminosities of the associated FIR sources between 20 and $10^{6} L_{\odot}$. This database provides the backbone for the discussion of the main long-term properties of maser emission that will appear in a forthcoming paper. We have identified several quantities that characterize the basic properties of water masers in star forming regions. These include:

(a) A plot of the time-velocity-flux density conveniently describes the morphology of the variability of the maser emission. This diagram is particularly useful to recognize the presence of possible velocity drifts, to separate steady components from bursts of short duration, and to study the properties of the emission features as a function of velocity.

(b) The integrated flux as a function of time describes the overall emission of the maser components associated with a star forming region. At present, we have not attempted to produce plots of the flux density as a function of time for individual velocity components. This type of presentation would produce an extremely high number of plots whose meaning and usefulness are not evident. The information, however, can be partly recovered from the time-velocity-flux density diagrams.

(c) The upper envelope of the maser emission is useful to determine the maximum emission that the star forming region could produce if all the velocity components were present simultaneously and emitting at their maximum rate. Similarly, the lower envelope pinpoints the steady components and their lowest level of emission.

(d) The statistical properties of the maser emission as a function of velocity are directly gauged by the distribution of the frequency of occurrence.

(e) The mean velocities derived from the upper envelope $\left(V_{\mathrm{up}}\right)$ and from the frequency of occurrence $\left(V_{\mathrm{fr}}\right)$ represent the velocity of the cluster of maser components and are suitable for comparison with the systemic velocity of the molecular cloud.

(f) Among the sources analyzed in this paper, Mon R2 IRS3 and G32.74-0.08 stand out as exceptional, in the sense that $\mathrm{H}_{2} \mathrm{O}$ maser emission is present at all times in a single component within a narrow velocity interval. In both cases, a velocity drift is present.

Acknowledgements. This long term project could not have been carried out without the help and dedication of the technical staff of the Radio Group of the Arcetri Observatory.

\section{References}

Akeson, R. L., \& Carlstrom, J. E. 1996, ApJ, 470, 528

Altenhoff, W. J., Downes, D., Pauls, T., \& Schraml, J. 1978, A\&AS, 35, 23

Anglada, G., Estalella, R., Pastor, J., Rodríguez, L. F., \& Hascick, A. D. 1996, ApJ, 463, 205

Aspin, C., \& Welther, D. M. 1990, A\&A, 235, 387

Aspin, C., Sandell, G., \& Walther, D. M. 1992, MNRAS, 258, 684

Bachiller, R., \& Cernicharo, J. 1986, A\&A, 166, 283

Bally, J., Devine, D., Alten, V., \& Sutherland, R. S. 1997, ApJ, 478, 603

Bechis, K. P., Harvey, P. M., Campbell, M. F., \& Hoffmann, W. F. 1978, ApJ, 226, 439 
Becker, R. H., White, R. L., \& Helfand, D. J. 1994, ApJS, 91, 347

Beckwith, S., Evans, N. J. II, Becklin, E. E., \& Neugebauer, G. 1976, ApJ, 208, 390

Berulis, I. I., Lekht, E. E., \& Mendoza-Torres, E. 1995, Astr. Rep., 39, 411

Berulis, I. I., Lekht, E. E., \& Rudnitskii, G. M. 1996, Astr. Rep., 40, 36

Bos, A. 1991, IEEE Trans. Instr. Meas., 40, 591

Brand, J., \& Blitz, L. 1993, A\&A, 275, 67

Brand, J., Cesaroni, R., Caselli, P., et al. 1994, A\&AS, 103, 541

Bronfman, L., Nyman, L.-A., \& May, J. 1996, A\&AS, 115, 81

Brown, A. T., Little, L. T., Macdonald, G. M., \& Matheson, D. N. 1982, MNRAS, 201, 121

Burton, M. G., Geballe, T. R., \& Brand, P. W. J. L. 1989, MNRAS, 238, 1513

Calamai, G., Felli, M., \& Giardinelli, S. 1982, A\&A, 109, 123

Carpenter, J. M., Meyer, M. R., Dougados, C., Strom, S. E., \& Hillenbrand, L. A. 1997, AJ, 114, 198

Carral, P., \& Welch, J. W. 1992, ApJ, 385, 244

Cesaroni, R. 1990, A\&A, 233, 513

Cesaroni, R., Palagi, F., Felli, M., et al. 1988, A\&AS, 76, 445

Cesaroni, R., Walmsley, C. M., Kömpe, C., \& Churchwell, E. 1991, A\&A, 252, 278

Cesarsky, C. J., Cesarsky, D. A., Churchwell, E., \& Lequeux, J. 1978, A\&A, 68, 33

Chernin, L. M., \& Masson, C. R. 1992, ApJ, 396, L35

Chernin, L. M., \& Masson, C. R. 1993, ApJ, 403, L21

Claussen, M. J., Wilking, B. A., Benson, P. J., et al. 1996, ApJS, 106, 111

Cohen, M. 1980, AJ, 85, 29

Comoretto, G., Palagi, F., Cesaroni, R., et al. 1990, A\&AS, 84, 179

Davis, C. J., Ray, T. P., Eislöffel, J., \& Corcoran, D. 1997a, A\&A, 324, 263

Davis, C. J., Eislöffel, J., Ray, T. P., \& Jenness, T. 1997b, A\&A, 324, 1013

Dent, W. A. 1972, ApJ, 177, 93

Dent, W. R. F., Little, L. T., Kaifu, N., Ohishi, M., \& Suzuki, S. 1985, A\&A, 146, 375

Eiroa, C., Palacios, J., \& Casali, M. M. 1998, A\&A, 335, 243

Eiroa, C., Casali, M. M., Miranda, L. F., \& Ortiz, F. 1994, A\&A, 290, 599

Eislöffel, J. 2000, A\&A, 354, 236

Felli, M., \& Churchwell, E. 1972, A\&AS, 5, 369

Fey, A. L., Gaume, R. A., Nedoluha, G. E., \& Claussen, M. J. 1994, ApJ, 435, 738

Fich, M. 1986, ApJS, 86, 475

Fich, M. 1993, AJ, 92, 787

Forster, J. R., \& Caswell, J. L. 1989, A\&A, 213, 339

Forster, J. R., \& Caswell, J. L. 1999, A\&AS, 137, 43

Frerking, M. A., \& Langer, W. D. 1982, ApJ, 256, 623

Fuente, A., Neri, R., Martín-Pintado, J., et al. 2001, A\&A, 366, 873

Fukui, Y. 1989, in ESO Workshop on Low-Mass Star Formation and Pre-Main sequence Objects, ed. B. Reipurth (Münich, ESO), 95

Garden, R. P., Russell, A. P. G., \& Burton, M. G. 1990, ApJ, 354,232

Gaume, R. A., Fey, A. L., \& Claussen, M. J. 1994, ApJ, 432, 648

Genzel, R., \& Downes, D. 1977, A\&AS, 30, 145
Giannakopoulou, J., Mitchell, G. F., Hasegawa, T. I., Matthews, H. E., \& Maillard, J.-P. 1997, ApJ, 487, 346

Gilmore, W. 1980, AJ, 85, 894

Girart, J. M., Ho, P. T. P., Rudolph, A. L., et al. 1999, ApJ, 522, 921

Goldsmith, P. F., Snell, R. L., Hemeon-Heyer, M., \& Langer, W. D. 1984, ApJ, 286, 599

Harker, D., Bregman, J., Tielens, A. G. G. M., Terni, P., \& Rank, D. 1997, A\&A, 324, 629

Harvey, P. M., Wilking, B. A., \& Joy, M. 1984, ApJ, 278, 156

Hashick, A. D., \& Ho, P. T. P. 1985, ApJ, 292, 200

Hayashi, M., Hasegawa, T., Omodaka, T., Hayashi, S. S., \& Miyawaki, R. 1987, ApJ, 312, 327

Hayashi, M., \& Murata, Y. 1992, PASJ, 44, 391

Heaton, B. D., \& Little, L. T. 1988, A\&A, 195, 193

Heaton, B. D., Little, L. T., \& Bishop, I. S. 1989, A\&A, 213, 148

Herbig, G. H., \& Jones, B. F. 1983, AJ, 88, 1040

Ho, P. T. P., Haschick, A. D., \& Israel, F. P. 1981, ApJ, 243, 526

Hodapp, K.-W. 1987, A\&A, 172, 304

Hodapp, K.-W. 1994, ApJS, 94, 615

Hofner, P., \& Churchwell, E. 1996, A\&AS, 120, 283

Howard, E. M., Pipher, J. L., \& Forrest, W. J. 1994, ApJ, 425, 707

Hunter, T. R., Taylor, G. B., Felli, M., \& Tofani, G. 1994, A\&A, 284, 215

Hunter, T. R., Churchwell, E., Watson, C., et al. 2000, AJ, 119, 2711

Israel, F. P. 1976, A\&A, 52, 175

Juan, J., Bachiller, R., Kömpe, C., \& Martín-Pintado, J. 1993, A\&A, 270, 432

Kutner, M. L., \& Tucker, K. D. 1975, ApJ, 199, 79

Ladd, E. F., Lada, E. A., \& Myers, P. C. 1993, ApJ, 410, 168

Lada, C. J., Blitz, L., Reid, M. J., \& Moran, J. M. 1981, ApJ, 243, 769

Lane, A. P., \& Bally, J. 1986, ApJ, 310, 820

Lekht, E. E. 1995, Astr. Rep., 39, 27

Lekht, E. E., Pashchenko, M. I., Rudnitskii, G. M., \& Sorochenko, R. L. 1982, Sov. Astr., 26, 168

Lekht, E. E., Likhachev, S. F., Sorochenko, R. L., \& Strelnitskij, V. S. 1993, AZh, 70, 731

Lekht, E. E., Mendoza-Torres, E., \& Sorochenko, R. L. 1995, Astr. Rep., 39, 34

Lekht, E. E., Mendoza-Torres, J. E., Pashchenko, M. I., \& Berulis, I. I. 1999, A\&A, 343, 241

Levreault, R. M. 1988, ApJS, 67, 283

Liljeström, T., \& Gwinn, C. R. 2000, ApJ, 534, 781

Liljeström, T., Mattila, K., Toriseva, M., \& Anttila, R. 1989, A\&AS, 79, 19

Liszt, H. S. 1995, AJ, 109, 1204

Liszt, H. S., Braun, R., \& Greisen, E. W. 1993, AJ, 106, 2349

Little, L. T., Brown, A. T., Riley, P. W., et al. 1983, MNRAS, 203, 409

Little, L. T., Dent, W. R. F., Heaton, B., Davies, S. R., \& White, G. J. 1985, MNRAS, 217, 227

Little, L. T., Kelly, M. L., \& Murphy, B. T. 1998, MNRAS, 294,105

Loren, R. B. 1977, ApJ, 215, 129

Loren, R. B. 1981, ApJ, 249, 550

Márquez, A., Mendoza-Torres, J. E., \& Lekht, E. E. 1998, A\&AS, 128, 1

Massi, M., Felli, M., \& Simon, M. 1985, A\&A, 152, 387 
Matthews, H. E., Little, L. T., Nyman, L.-A., \& Macdonald, G. H. 1984, A\&A, 136, 282

Matthews, H. E., Olnon, F. M., Winnberg, A., \& Baud, B. 1985, A\&A, 149, 227

Meehan, L. S. G., Wilking, B. A., Claussen, M. J., Mundy, L. G., \& Wootten, A. 1998, AJ, 115, 1599

Megeath, S. T., \& Wilson, T. L. 1997, AJ, 114, 1106

Minchin, N. R., White, G. J., \& Padman, R. 1993, A\&A, 277, 595

Moriarty-Schieven, G. H., Snell, R. L., \& Hughes, V. A. 1989, ApJ, 347, 358

Palagi, F., Cesaroni, R., Comoretto, G., Felli, M., \& Natale, V. 1993, A\&AS, 101, 153

Palla, F., Brand, J., Comoretto, G., Felli, M., \& Cesaroni, R. 1991, A\&A, 246, 249

Pankonin, V., Winnberg, A., \& Booth, R. S. 1977, A\&A, 58, L25

Persi, P., Palagi, F., \& Felli, M. 1994, A\&A, 291, 577

Persi, P., Ferrari-Toniolo, M., Shivanandan, K., \& Spinoglio, L. 1989, A\&AS, 70, 437

Persson, S. E., Geballe, T. R., Simon, T., Lonsdale, C. J., \& Baas, F. 1981 ApJ, 251, L85

Phillips, J. P., White, G. J., Rainey, R., et al. 1988, A\&A, 190, 289

Pismis, P., \& Mampaso, A. 1991, MNRAS, 249, 385

Plume, R., Jaffe, D. T., \& Evans II, N. J. 1992, ApJS, 78, 505

Plume, R., Jaffe, D. T., Evans II, N. J., Martín-Pintado, J., \& Gómez-Gonzáles, J. 1997, ApJ, 476, 730

Racine, R. 1968, AJ, 73, 233

Ray, T. P., Poetzl, R., Solf, J., \& Mundt, R. 1990, ApJ, 357, L45

Rodríguez, L. F., Moran, J. M., Ho, P. T. P., \& Gottlieb, E. W. 1980, ApJ, 235, 845

Sandell, G., \& Olofsson, H. 1981, A\&A, 99, 80

Sandell, G., Nyman, L. A., Winnberg, A., \& Hascick, A. 1985, in Nearby Molecular Clouds, ed. G. Serra (Springer: Berlin), Lect. Notes Phys., 237, 234

Schwartz, P. R. 1989, ApJ, 338, L25

Snell, R. L., \& Bally, J. 1986, ApJ, 303, 683
Snell, R. L., Scoville, N., Sanders, D. B., \& Erickson, N. R. 1984, ApJ, 284, 176

Strom, S. E., Grasdalen, G. L., \& Strom, K. M. 1974, ApJ, 191,111

Tafalla, M., Bachiller, R., \& Martín-Pintando, J. 1993, ApJ, 403, 175

Tafalla, M., Bachiller, R., \& Wright, M. C. H. 1994, ApJ, 432, L127

Tafalla, M., Bachiller, R., Wright, M. C. H., \& Welch, W. J. 1997, ApJ, 474, 329

Tapia, M., Persi, P., Bohigas, J., \& Ferrari-Toniolo, M. 1997, AJ, 113, 1769

Tofani, G., Felli, M., Taylor, G. B., \& Hunter, T. R. 1995, A\&AS, 112, 299

Torrelles, J. M., Gómez, J. F., Rodríguez, L. F., Curiel, S., \& Anglada, G. 1998, ApJ, 505, 756

Thronson, H. A., Jr., Gatley, I., Harvey, P. M., Sellgren, K., \& Werner, M. W. 1980, ApJ, 237, 66

Valdettaro, R., Palla, F., Brand, J., et al. 2001a, A\&A, 368, 845

Valdettaro, R., Palla, F., Brand, J., et al. 2001b, in Cosmic Masers, ed. V. Migenes (San Francisco: ASP Conf. Ser.), in press

Walther, D. M., Aspin, C., \& McLean, I. S. 1990, ApJ, 356, 544

Walther, D. M., Geballe, T. R., \& Robson, E. I. 1991, ApJ, 377,246

Wilner, D. J., \& Welch, W. J. 1994, ApJ, 427, 898

Wolf, G. A., Lada, C. J., \& Bally, J. 1990, AJ, 100, 1892

Wood, D. O. S., \& Churchwell, E. 1989, ApJ, 340, 265

Wouterloot, J., \& Brand, J. 1989, A\&AS, 80, 149

Wouterloot, J. G. A., Fiegle, K., Brand, J., \& Winnewisser, G. 1995, A\&A, 301, 236 (Erratum in 1997, A\&A, 319, 360)

Zhou, S., Evans, N. J. II, Mundy, L. G., \& Kutner, M. L. 1993, ApJ, 417, 613

Zinchenko, I., Pirogov, L., \& Toriseva, M. 1998, A\&AS, 133, 337

Zoonematkermani, S., Helfand, D. J., Becker, R. H., White, R. L., \& Perley, R. A. 1990, ApJS, 74, 181 